\title{
STRAF- UND BUßGELDRECHTLICHE VERANTWORTUNG VON UNTERNEHMEN, INSBESONDERE IM KARTELL-, DATENSCHUTZ- UND BANKENAUFSICHTSRECHT, DE LEGE LATA UND DE LEGE FERENDA
}

\author{
Criminal and administrative responsibility of enterprises, especially \\ in the area of cartel law, data protection law and banking supervision
}

\author{
GERHARD DANNECKER*
}

\begin{abstract}
In the law of the European Union, liability for (administrative) fines (Geldbußen) has developed as a special sanction model for regulatory offences, first in the area of cartel law, now of data protection and banking supervisiory law, too. The criminal law systems for judicial persons in the EU member states are oriented towards the EU system of enterprise liability. This leads to problems, especially with regard to the sentencing and to procedural guarantees. The emerging path of convergence despite different sanction models - responsibility of enterprises versus responsability of judicial persons - should be continued in view of the punitive nature of the (administrative) fine.
\end{abstract}

Keywords: Corporate fines, criminal liability of legal persons, antitrust fines, data protection fines, banking supervisory fines

\section{EiNLEITUNG}

Von der Strafrechtswissenschaft weitgehend unbemerkt hat sich im europäischen Kartellrecht ein Sanktionsinstrument entwickelt, das als Bußgeldrecht gegen Unternehmen umschrieben werden kann. ${ }^{1}$ Im Wettbewerbsrecht der Europäischen Union wird unter dem Begriff des Unternehmens nach ständiger Rechtsprechung des EuGH ,jede eine wirtschaftliche Tätigkeit ausübende Einheit unabhängig von ihrer Rechtsform sowie der Art ihrer Finanzierung "2, also die wirtschaftliche Einheit, verstanden, unabhängig davon, ob diese nach nationalem Recht Rechtspersönlichkeit aufweist.

* Prof. Dr. Dr. h.c. Seniorprofessor, Institut für deutsches, europäisches und internationales Strafrecht und Strafprozessrecht, Juristische Fakultät, Universität Heidelberg, Universität Miskolc.

1 Eingehend dazu DANNECKER, Gerhard - SCHRÖDER, Thomas: Tatbestände mit supranationaler Schutzrichtung (Europadelikte). In: BÖSE, Martin (Hrsg.): Europäisches Strafrecht (EnzEuR Bd. 11). 2. Aufl., 2021, § 8 Rn. 246 ff.; DANNECKER, Gerhard - DANNECKER, Christoph: Europäische und verfassungsrechtliche Vorgaben für das materielle und formelle Unternehmensstrafrecht. NZWiSt, 2016, $162 \mathrm{ff}$.

2 EuGH, 23. 04. 1991, Rs. C-41/90, Slg. 1991, I-2010, Rn. 21 (Höner und Elser); EuGH, 17. 02. 1993, verb. Rs. C-159/91 und C-160/91, Slg. 1993, I-664, Rn. 17 (Poucet et Pistre); EuGH, 10. 09. 2009, Rs. C-97/08 P, Slg. 2009, I-8237, Rn. 54 (Akzo Nobel). 
Die bußgeldrechtliche Verantwortung von Unternehmen stellt einen Fremdkörper im kontinentaleuropäischen Strafrechtsdenken dar. Er entstammt dem US-amerikanischen Recht und wurde von der Europäischen Union übernommen, als diese im Jahr 1962 die bußgeldrechtliche Verantwortung von Unternehmen mit der Verordnung 17/62 ${ }^{3}$ eingeführt hat. Das Kartellbußgeldrecht der Europäischen Union gehört zu den klassischen Bereichen eines eigenständigen europäischen Sanktionenrechts, das die Kommission unter der Kontrolle des EuGH in effektiver Weise implementiert hat. ${ }^{4}$ Zuständig für die Verhängung der unionsrechtlichen Geldbußen ist allein die Europäische Kommission; die Wettbewerbsbehörden der Mitgliedstaaten können auf der Grundlage der europäischen Bußgeldregelungen keine Sanktionen verhängen. Im Rahmen der Dezentralisierung der kartellrechtlichen Rechtsanwendung, die im Jahr 2003 auf Unionsebene eingeführt wurde, können und müssen die Wettbewerbsbehörden der Mitgliedstaaten nun auch - auf der Grundlage ihrer nationaler Sanktionsvorschriften - Wettbewerbsverstöße sanktionieren.

Nach der Ausweitung des unionsrechtlichen Bußgeldrechts gegen Unternehmen betrifft dieses nunmehr drei Bereiche, auf die im Folgenden näher eigegangen werden soll:

(1.) Das kartellrechtliche Unternehmensbußgeldrecht ist das am weitesten entwickelte Sanktionssystem der Europäischen Union mit strafrechtlichem Charakter. ${ }^{5}$ Die Mitgliedstaaten waren aufgrund der ECN+-Richtlinie ${ }^{6}$ verpflichtet, bis zum 04. 02. 2021 das unionsrechtliche Konzept der bußgeldrechtlichen Verantwortung von Unternehmen für Kartellrechtsverstöße in ihre nationalen Rechtsordnungen zu übernehmen.

3 Erste DurchführungsVO zu den Art. 85 und 86 des Vertrages vom 6. 2. 1962, AB1. 13 vom 21. 2. 1962, p. 204.

4 Eingehend dazu DANNECKER, Gerhard - FISCHER-FRITSCH, Jutta: Das EG-Kartellrecht in der Bußgeldpraxis. 1989, passim; DANNECKER, Gerhard: Europäisches Kartellstrafrecht und internationale Tendenzen. In: SCHICK, Peter J. - HILF, Marianne: Kartellstrafrecht. Das Spiel vom Fragen (P. Handke). Wien, 2007, 31 ff.; zusammenfassend DANNECKER, Gerhard - DANNECKER, Christoph: Kartellsanktionsrecht. In: SOYER, Richard (Hrsg.): Handbuch Unternehmensstrafrecht. Wien, 2021, § 18 Rn. 18.13 ff.

5 Generalanwältin Kокотт, Juliane: Schlussanträge vom 28. 2. 2013 in der Rechtssache C681/1 Rn. 40; Generalanwalt LÉGER, Philippe: Schlussanträge vom 3. 2. 1998 in der Rechtssache C-185/95 P - Baustahlgewerbe, EuGH Slg. 1998, I-8417 Rn. 30 Fn. 25; eingehend dazu DANNECKER - FISCHER-FRITSCH: a.a.O. 6 f.; siehe auch DANNECKER DANNECKER (2021): a.a.O. $§ 8$ Rn. 18.37 ff. mit weit. Nachw.; NowAK, Carsten: In: LOEWENHEIM, Ulrich - MEESEN, Karl M. - RIESENKAMPFF, Alexander - KERSTING, Christian - MEYER-LindEMANN, Hans Jürgen (Hrsg.): Kartellrecht. Kommentar zum Deutschen und Europäischen Recht. 4. Aufl., 2020, München. Art. 23 VerfVO Rn. 50; TIEDEMANN, Klaus: Der Allgemeine Teil des europäischen supranationalen Strafrechts. In: Festschrift für Hans-Heinrich Jescheck zum siebzigsten Geburtstag. 1985, 1411 und 1417.

6 RL 2019/1/EU des Europäischen Parlaments und des Rates vom 11. 12. 2018 zur Stärkung der Wettbewerbsbehörden der Mitgliedstaaten im Hinblick auf eine wirksamere Durchsetzung der Wettbewerbsvorschriften und zur Gewährleistung des reibungslosen Funktionierens des Binnenmarkts, AB1. L11/3 (14. 1. 2019). 
Auf diese Weise soll das Ziel einer Harmonisierung der Kartellsanktionsrechtssysteme der Union und ihrer Mitgliedstaaten erreicht werden.

(2.) Auch das Datenschutzrecht kennt inzwischen Unternehmensgeldbußen. ${ }^{7}$ Diese Sanktionssysteme hat die Europäische Union unter Rückgriff auf die Unternehmensverantwortung im Kartellbereich für den Bereich des Datenschutzrechts mit der Datenschutz-Grundverordnung ${ }^{8}$ eingeführt. Diese Unternehmensgeldbußen sind ausschließlich von den zuständigen nationalen Behörden zu verhängen.

(3.) Auch für den Bereich des Bankenaufsichtsrechts existieren unionsrechtliche Bußgeldregelungen, die sich gegen juristische Personen und gegen Unternehmen richten. Zuständig für die Verhängung der Sanktionen sind die Europäische Zentralbank und die Zentralbanken der Mitgliedstaaten, je nach Zuständigkeit für die Bankenaufsicht. Diese Sanktionen gegen Unternehmen werden in der Praxis regelmäßig angewendet, wenn im Rahmen der Bankenaufsicht Rechtsverstöße festgestellt werden.

(4.) In Deutschland befand sich der Entwurf eines Verbandsverantwortlichkeitsgesetzes im Gesetzgebungsprozess, ${ }^{9}$ der allerdings in der laufenden Legislaturperiode nicht mehr verabschiedet wurde und deshalb der Diskontinuität unterfiel. Der Gesetzentwurf sah die Einführung der Strafbarkeit juristischer Personen und sonstiger Verbände vor. Vorgesehen war, dass im Falle einer Straftat durch eine Leitungsperson der Verband hierfür sanktioniert werden kann. Gleichwohl wurden einzelne Elemente des Modells der Unternehmenssanktionierung aufgegriffen. Dadurch sollte die bisherige bußgeldrechtliche Verantwortung juristischer Personen und sonstiger Verbände durch ein strafrechtliches Sanktionssystem abgelöst werden, das durch Einbeziehung der Verantwortung einer Muttergesellschaft und durch die Bestimmung des Sanktionsrahmens nach dem Umsatz der wirtschaftlichen Einheit dieselbe Reichweite und Sanktionshöhe wie das unternehmensbußgeldrechtliche Modell erreichen soll. Ob dieses Gesetzesvorhaben in der neuen Legislaturperiode wieder aufgegriffen wird, ist gegenwärtig nicht abzusehen.

\section{ENTWICKLUNG DES KARTELLBUBGELDRECHTS GEGEN UNTERNEHMEN IN DER EUROPÄISCHEN UNION}

Der Wettbewerb stellt das leitende Grundprinzip der europäischen Wirtschaftsverfassung dar, die sich in Art. 4 Abs. 1 EG zum Grundsatz einer offenen Marktwirtschaft mit freiem Wettbewerb bekennt. Der Wettbewerb gilt als Motor höchster ökonomischer Leistungsfähigkeit bei größtmöglicher Freiheitssicherung. ${ }^{10} \mathrm{Ihm}$

7 Dazu DANNECKER, Gerhard - SCHRÖDER, Thomas: a.a.O. § 8 Rn. 313 ff., 325 ff.

8 Verordnung (EU) 2016/679 des Europäischen Parlaments und des Rates vom 27. April 2016 zum Schutz natürlicher Personen bei der Verarbeitung personenbezogener Daten, zum freien Datenverkehr und zur Aufhebung der Richtlinie 95/46/EG, ABl. L 119 vom 4. 5. 2016, 1, berichtigt ABl. L 314 vom 22. 11. 2016, 72, ABl. L 127 vom 23. 5. 2018, 2.

9 Bundesgesetz über die Verantwortlichkeit von Verbänden für Straftaten (Verbandsverantwortlichkeitsgesetz - VbVG), BGBl. I Nr. 151/2005.

10 Näher dazu HoPPMANN, Erich: Das Konzept der optimalen Wettbewerbsintensität, JbNSt, 179 (1966), 286, 289 und 300. 
werden vor allem wirtschaftspolitische, daneben aber auch gesellschaftspolitische Funktionen zugeschrieben. Deshalb wurde der Europäischen Kommission für diesen Bereich die Kompetenz eingeräumt (Art. 103 AEUV), Bußgeldtatbestände gegen Unternehmen einzuführen, um ein unionsrechtliches Sanktionssystem zur Durchsetzung des Kartellrechts zu schaffen. Dadurch soll der Wettbewerb als Rechtsgut geschützt werden. ${ }^{11}$

\subsection{Entwicklung des unionsrechtlichen Bußgeldrechts gegen Unternehmen}

Die Europäische Union hat von ihrer Kompetenz, für das Gebiet des Kartellrechts Bußgeldtatbestände gegen Unternehmen einzuführen, im Jahr 1962 Gebrauch gemacht und die Verordnung 17/62 ${ }^{12}$ eingeführt. Deren Art. 15 sah für schuldhafte vorsätzliche und fahrlässige - Zuwiderhandlungen sowohl gegen Mitwirkungspflichten als auch gegen das Kartell- und Missbrauchsverbot der Art. 81, 82 EG die Verhängung gemeinschaftsrechtlicher Geldbußen vor. Damit stellte das EG-Kartellrecht das am weitesten entwickelte Sanktionssystem des Gemeinschaftsrechts bereit, das zunächst ausschließlich von der Kommission (nicht auch von den Mitgliedstaaten) angewendet werden konnte. Damit war eine einheitliche Rechtslage auf der Ebene der Europäischen Union gewährleistet, für deren Anwendung ausschließlich die Generaldirektion IV der Europäischen Kommission zuständig war. Die Mitgliedstaaten der Europäischen Union kannten eigene kartellrechtliche Sanktionssysteme, die nur auf innerstaatliche Kartellrechtsverstöße anwendbar waren; diese nationalen Sanktionssysteme wiesen erhebliche Unterschiede auf. ${ }^{13}$ Diesbezüglich bestanden damals keine Harmonisierungsbestrebungen.

\subsection{Charakteristika des europäischen Kartellbußgeldrechts}

Fragt man nach den für die Geldbußen gegen Unternehmen charakteristischen Eigenschaften des europäischen Kartellordnungswidrigkeitenrechts, so sind folgende Aspekte hervorzuheben:

(1.) Bußgeldentscheidungen gemäß Art. 23 Abs. 5 VO 1/2003 sind „Entscheidungen nicht strafrechtlicher Art", doch sind auch diese Geldbußen dem Strafrecht im weiteren Sinne zuzuordnen. ${ }^{14}$ Denn die Geldbußen besitzen vor allem eine repressive

11 DANNECKER, Gerhard: Der strafrechtliche Schutz des Wettbewerbs: Notwendigkeit und Grenzen einer Kriminalisierung von Kartellrechtsverstößen. In: Festschrift für Klaus Tiedemann zum 70. Geburtstag. Köln, 2008, 789 ff.

12 Erste DurchführungsVO zu den Art 85 und 86 des Vertrages vom 6. 2. 1962, ABl. 13 vom 21. 2. 1962, 204.

13 Näher dazu DANNECKER, Gerhard - KÖRTEK, Jasemin: General Report. In: DANNECKER, Gerhard - JANSEN, Oswald (eds.): Competition Law Sanctioning in the European Union. 2004, 1 ff; WAGNER VON PAPP, Florian: Kartellstrafrecht in den USA, dem Vereinigten Königreich und Deutschland. $W u W, 2009,1236$ ff.

14 Vgl. BIERMANN, Jörg: In: IMMENGA, Ulrich - MESTMÄCKER, Ernst Joachim (Hrsg.): Wettbewerbsrecht. Bd. 1, 6. Aufl., 2020, Vor Art 23 VO 1/2003 Rn. 39 mit weit. Nachw.; kritisch dazu WILS, Wouter P. J.: World Competition. 2005, 5 ff. 
Funktion: ${ }^{15}$ Die Verhängung gemäß Art. 23 Abs. 1 VO 1/2003 setzt vorsätzliches oder fahrlässiges Verhalten voraus, weshalb die Geldbuße eine Reaktion auf rechtswidriges Verhalten darstellt. Auf strafähnliche Maßnahmen, die repressive Zwecke verfolgen, sind unstreitig die strafrechtlichen Garantien anzuwenden. ${ }^{16}$ Die Schwere der angedrohten Sanktion stellt ein weiteres Indiz für den strafrechtlichen Charakter der EU-Bußgelder dar. ${ }^{17}$ Aus diesem Grund wird zwischen Kriminalstrafen und Geldbußen als strafrechtlichen Sanktionen im weiteren Sinne, vergleichbar dem deutschen, italienischen und portugiesischen Bußgeldrecht ${ }^{18}$, differenziert. Entsprechend verweist die Kommission für die Unternehmensgeldbußen auf die das Strafrecht betreffenden Grundrechte und insbesondere auf die im Strafrecht Geltung beanspruchenden Prinzipien, die in der Grundrechtecharta niedergelegt sind oder sich aus der EMRK $^{19}$ und den mitgliedstaatlichen Verfassungstraditionen ableiten lassen. ${ }^{20}$

(2.) Das europäische Kartellbußgeldrecht richtete sich von Anfang an ausschließlich gegen Unternehmen und Unternehmensvereinigungen und nicht gegen juristische Personen und sonstige Verbände. ${ }^{21}$ Das nationale Rechtsträgerprinzip, das in den Mitgliedstaaten der Europäischen Union ganz überwiegend gilt und zur Verneinung der Rechtsträgerschaft von Unternehmen führt, kommt im EU-Kartellrecht nicht

$15 \mathrm{Zu}$ den Funktionen der Geldbuße siehe BIERMAnN, Jörg: In: IMMENGA - MESTMÄCKER (Hrsg.): Wettbewerbsrecht. Bd. 1, Vor Art 23 VO 1/2003 Rn. 22 ff.

16 EuGH, 8. 7. 1999, Rs. C-199/92 P, Slg 1999, I-4384 (Hüls); 8.7.1999, Rs. C-199/92 P, Slg 1999, I-4631 (Montecatini); RIZVI, Salim: Entfesselte Bussenpraxis im Wettbewerbsrecht? AJP/PJA, 2010, 452 und 457 f; FEDERMANN, Bernd A.: Kriminalstrafen im Kartellrecht. 2006, 92; DANNECKER, Gerhard: Stellungnahme zum Zwischenbericht des Bundeskartellamts zum Expertenkreis Kartellsanktionenrecht. NZKart, 2015, $14 \mathrm{f}$.

17 DANNECKER - FISCHER-FRITSCH: a.a.O. 352; MÖHLENKAMP, Andreas: Die europäische Bußgeldpraxis aus Unternehmenssicht in: SCHWARZE, Jürgen (Hrsg.), Instrumente zur Durchsetzung des europäischen Wettbewerbsrechts, 2002, 121, 125; Wegner, Carsten: Keine umfassende Begründungspflicht der Kommission für Geldbußen in Millionenhöhe? WuW 2001, 469, 476.

${ }^{18}$ DANNECKER - FISCHER-FRITSCH: a.a.O. 6 f.; TIEDEMANN, Klaus: Der Allgemeine Teil des europäischen supranationalen Strafrechts. In: Festschrift für Hans-Heinrich Jescheck zum siebzigsten Geburtstag. 1985, 1411 und 1417.

19 Die Rechtsprechung bedient sich seit einem Urteil des EGMR (8. 6. 1976, 5100/71 ua, Rz 83ff [Engel ua] = EuGRZ 1976, 221) aus dem Jahr 1976 dreier Kriterien für die Annahme des Vorliegens einer strafrechtlichen Anklage („Engel-Kriterien“): der Zuordnung einer Vorschrift im nationalen Recht, der Natur des Vergehens sowie der Art und Schwere der Sanktion.

20 Siehe Charta der Europäischen Union vom 7.12.2000 in der am 12. 12. 2007 angepassten Fassung (ABl. C 83 v. 30. 3. 2010, 389 ff); EuG 27. 9. 2006, T-43/02, Rz 74, Slg 2006 II3435 (Jungbunzlauer); 8. 7. 2008, T-99/04, Rz 45, Slg 2008 II-1501 (AC Treuhand); SAUER, Ralf: In: SCHULTE, Lothar - JUST, Christoph: Kartellrecht. ${ }^{2}$ Art 23 VO 1/2003 $\mathrm{Rz} 40 \mathrm{ff}$.

21 Eingehend dazu SCHILDGEN, Larissa: Rechtsfähigkeit des Unternehmens im Unionswettbewerbsrecht. 2020, $7 \mathrm{ff}$. 
zum Tragen. Dort wurde Unternehmen durch den Unionsgesetzgeber mit der Einführung der Verantwortung der Unternehmen zugleich die Eigenschaft einer Rechtsperson verliehen. ${ }^{22}$

(3.) Die für das Unternehmen handelnden natürlichen Personen können nach dem Unionsbußgeldrecht nicht für Kartellverstöße geahndet werden. Ihr Verhalten wird den Unternehmen in sehr weitgehendem Umfang zugerechnet; es ist nicht erforderlich, dass eine Leitungsperson den Rechtsverstoß begangen hat. Eine Ahndung der natürlichen Personen für Verstöße gegen das Unionskartellrecht ist nur nach nationalem Recht und dem dort geltenden Sanktionsregime möglich.

(4.) Der allgemeine Teil des europäischen Bußgeldrechts ist in weiten Teilen nicht gesetzlich geregelt. Er wurde aus allgemeinen Rechtsgrundsätzen unter Rückgriff auf die Rechtsordnung der Mitgliedstaaten entwickelt und kennt bis heute nur für einige Fragen, so zB. für den Irrtum und die Verjährung, spezielle Regelungen. ${ }^{23}$

(5.) Geldbußen können bei schwerwiegenden Kartellverstößen (Kartellabsprachen und Missbrauch einer marktbeherrschenden Stellung) bis zu 10\% des Gesamtumsatzes der wirtschaftlichen Einheit, der im Jahr vor der Bußgeldverhängung erzielt worden ist, verhängt werden. Die 10\%-Grenze stellt nach Auffassung des EuGH eine Kappungsgrenze dar. ${ }^{24}$ Dies bedeutet, dass als Ausgangspunkt der Bußgeldbemessung die 10\%-Grenze überschritten werden darf. Erst nach Berücksichtigung sämtlicher Milderungsgründe darf die 10\%-Grenze nicht mehr überschritten werden. Ist dies der Fall, so führt die 10\%-Grenze zu einer Kappung der zu verhängenden Geldbuße bei $10 \%$ des Konzernumsatzes.

(6.) Die Kommission hat in Anlehnung an das US-amerikanische Recht Sentencing Guidelines eingeführt ${ }^{25}$, um Transparenz und Objektivität der Entscheidungen der Kommission im Bereich der Geldbußen sowohl gegenüber dem Unternehmen als auch gegenüber dem Europäischen Gerichtshof, der die Rechtskontrolle ausführt, zu erhöhen. Durch die Leitlinien wurde die Bußgeldbemessung der Europäischen Kommission auf eine neue Grundlage gestellt.

(7.) Die Kommission hat eine eigenständige Kronzeugenregelung für die Mitwirkung bei der Aufdeckung von Kartellrechtsverstößen eingeführt. ${ }^{26}$ Vorbild hierfür

22 DANNECKER - DANNECKER: NZWiSt, 2016, 162, 166; ebenso HEINICHEN, Christian: Unternehmensbegriff und Haftungsnachfolge im europäischen Kartellrechts. Baden-Baden 2011, 76 ff., 154 ff.; vgl. auch LESSENICH, Christof A.: Unternehmensbegriff und Zurechnung. Bonn 2000, 35 ff., 99. A.A. HENN, Florian: Strafrechtliche Verfahrensgarantien im europäischen Kartellrecht. Implikationen und Grenzen der Strafrechtsähnlichkeit von Kartellbußgeldern. 2018, 33. Unternehmen als „Sanktionsobjekte für Kartellbußgelder“.

23 Näher dazu DANNECKER - SCHRÖDER: a.a.O. § 8 Rn. § 8 Rn. 279 ff.; STUCKENBERG, CarlFriedrich: Allgemeiner Teil eines Europäischen Strafrechts, in: BÖSE, MARTIN (Hrsg.): Europäisches Strafrecht (EnzEuR Bd. 11). 2. Aufl., 2021, § 10 Rn. 1 ff.

24 EuGH, 28.6.2005, verb. Rs C-189/02 P, C-202/02 P, C-205/02 P bis C-213/02 P, Slg. 2005, I-5488, Rn. 278 (Dansk Rørindustri); VON LAUFENBERG, Christoph: Kartellrechtliche Konzernhaftung. Berlin, 2018, 56.

25 Siehe dazu nur DANNECKER - DANNECKER (2021): a.a.O. § 18 Rn. 18,64 ff.

26 Siehe dazu nur DANNECKER - DANNECKER (2021): a.a.O. § 18 Rn. 18,67 ff. 
war die „Leniency Policy“ des US-Justizministeriums. ${ }^{27}$ Durch diese Mitteilung sollen größere Anreize für die Aufdeckung von Kartellen geschaffen werden; gleichzeitig soll die präventive Wirkung der Bußgelder erhöht werden, weil Unternehmen erfahrungsgemäß aufgrund der generellen Gefahr, dass ein Kartellant in der Zukunft einen „Kartellverrat" begehen wird, von vornherein von der Beteiligung an einem Kartell zurückschrecken. ${ }^{28}$ Es handelt sich also um ein an der wirksamen Aufdeckung und Verfolgung von Kartellen orientiertes Anreizsystem. ${ }^{29}$

(8.) Das Verfahren zur Verhängung der Bußgelder wird als Verwaltungsverfahren und nicht als strafrechtliches Verfahren geführt ${ }^{30}$, selbst wenn Geldbußen und damit Strafsanktionen im weiteren Sinne verhängt werden. ${ }^{31}$ In diesem Verwaltungsverfahren kommen gleichwohl einzelne strafprozessuale Garantien zur Anwendung ${ }^{32}$, so insbesondere der Grundsatz „ne bis in idem“. ${ }^{33}$ Hingegen soll der Grundsatz „,nemo tenetur" keine Anwendung finden. ${ }^{34}$

(9.) Die strafrechtlichen Sanktionen (im weiteren Sinne) des nationalen Rechts wegen Kartellrechtsverstößen gegen Art. 101 und 102 AEUV sind von den nationalen Sanktionsinstanzen anzuwenden. Das Verfahrensrecht in den Mitgliedstaaten der Europäischen Union muss rechtsstaatlich ausgestaltet $\operatorname{sein}^{35}$ und den unionsrechtlichen Verfassungsgarantien Rechnung tragen. Hierbei ist von einem integralen Rechtsstaatsverständnis auszugehen, nach dem sich das Rechtsstaatsprinzip nicht in

27 Vgl hierzu LuTZ, Martin: Amnestie für aufklärungsbereite Kartellanten? BB, 2000, 677 f.

2 KOM, Pressemitteilung zur Kronzeugenregelung, IP/02/274 vom 13. 2. 2002, 3.

29 Schlussanträge Generalanwalt MAZÁK, Ján v. 16. 12. 2010, C-360/09, Pfleiderer, Rz 31; EuG 9. 9. 2011, T-12/06, Rz 105 ff und 110 (Deltafina).

30 BIERMANN: In: IMMENGA - MESTMÄCKER (Hrsg.): Wettbewerbsrecht. Bd. 1, Vor Art. 23 VO 1/2003 Rn. 214.

31 EuGH, 13. 7. 1966, Rs. C-56/64 und 58/64, Slg. 1966, 299, 321 und 385 f. (Grundig); 15. 7. 1970, Rs. C-44/69, Slg 1970, 733 und 756 (Buchler); 18. 5. 1982, Rs. 155/79, Slg 1982, 1575 und 1611 (AM\&S); jeweils noch zur VO 17/62.

32 DANNECKER - DANNECKER (2021): a.a.O. § 18 Rn. 18.80.

33 BIERMANN: In: IMMENGA - MESTMÄCKER (Hrsg.): Wettbewerbsrecht. Bd. 1, Vor Art. 23 VO 1/2003 Rn. 242 ff; DANNECKER, Gerhard: Ne bis in idem und das Verbot straf- und kartellrechtlicher Prallelverfahren. 2021, 37 ff; DANNECKER, Gerhard: Grundstrukturen des Grundsatzes „ne bis in idem“ im Spiegel der Rechtsprechung des Europäischen Gerichtshofs für Menschenrechte und des Europäischen Gerichtshofs. In: Festschrift für Ákos Farkas zum 65. Geburtstag. Bd. 1, 2019, 206 ff. (https://www.mjsz.uni-miskolc.hu/files/6553/23_danneckergerhard_t\%C3\%Bórdelt.pdf); ESSER, Robert: Das Doppelverfolgungsverbot in der Rechtsprechung des EGMR Das Doppelverfolgungsverbot in der Rechtsprechung des EGMR (Art. 4 des 7. ZP EMRK). Divergenzen und Perspektiven. In: $\mathrm{HoCH}-$ MAYR, Gudrun (Hrsg.): Ne bis in idem in Europa. Baden-Baden 2015, 27 ff.

34 Kritisch dazu DANNECKER, Christoph: Konturierung prozessualer Gewährleistungsgehalte des nemo tenetur- Grundsatzes anhand der Rechtsprechung des EGMR. ZStW, 127 (2015), 370 ff.; HENN: a.a.O. 172 ff.

35 Zur Geltung strafrechtlicher Fundamentalgrundsätze DANNECKER - SCHRÖDER, Thomas: a.a.O. $§ 8$ Rn. 284 mit weit. Nachw.; HENN: a.a.O. 34. 
Einzelbestimmungen erschöpft. ${ }^{36}$ Rechtsstaatlichkeit ist auch als „Grundlage für $[\ldots]$ nicht erwähnte - unbenannte - Einzelgewährleistungen“ heranzuziehen. ${ }^{37} \mathrm{Di}$ esbezüglich weicht das Rechtsstaatsprinzip von der im anglo-amerikanischen Recht geltenden Rule of Law, die auf der Verwirklichung von Einzelgewährleistungen beruht, ab.

(10.) In den USA werden extrem hohe Sanktionen (fines) gegen Unternehmen angedroht, ohne dass zwischen Kriminalstrafen und Geldbußen als Strafen im weiteren Sinn differenziert wird. Die nach dem Gesetz vorgesehenen „fines“ enthalten ein so hohes Drohpotential, dass die Unternehmen de facto gezwungen sind, die zu verhängenden Sanktionen auszuhandeln. Demgegenüber kommt in der Europäischen Union und ihren Mitgliedstaaten dem konsensualen Erledigungsverfahren kein vergleichbarer Stellenwert zu, weil die zu verhängenden Sanktionen schuldangemessen sein müssen. Die Geldbußen werden von den zuständigen Behörden unter der Kontrolle der Gerichte verhängt, auch wenn es zu keiner Einigung mit den Unternehmen gekommen ist. Schuldunangemessene Geldbußen werden aufgehoben und angemessene Sanktionen von der Gerichten verhängt.

\subsection{Entwicklung des Kartellsanktionsrechts in der Europäischen Union}

Der EWG-Vertrag ermächtigte in Art. 83 Abs. 2 lit. a iVm Abs. 1 EG den Rat zur Einführung von Geldbußen und Zwangsgeldern durch entsprechende Verordnungen. Hiervon machte der Rat im Zusammenhang mit der Einführung der Verordnung 17/62 Gebrauch und sah in Art. 15 dieser Verordnung Unternehmensbußgelder vor.

\section{a. Einführung unionsrechtlicher Unternehmensgeldbußen durch die VO 17/62}

Die Kommission verfolgte mit den Bußgeldern erklärtermaßen sowohl repressive als auch präventive Ziele ${ }^{38}$; im Vordergrund stand die negative Generalprävention iSd Abschreckung, die vor allem durch immer höhere Geldbußen erreicht werden soll. ${ }^{39}$ Das einseitige Abstellen auf die Abschreckungswirkung führte vor allem bei der Bestimmung der Bußgeldhöhe häufig zu fragwürdigen Ergebnissen. ${ }^{40}$ Auch die Kommission räumt der positiven Generalprävention iSd Bestätigung der Rechtsordnung eine wichtige Bedeutung bei der Verhängung von Geldbußen ein.

Ein weiteres Ziel, das die Kommission mit der Verhängung der Bußgelder von Anfang an verfolgt hat, lag in der Gewinnabschöpfung. Auf der Rechtsgrundlage des

36 GRZESZICK, Bernd: In MAUnZ, Theodor/DÜRIG, Günter (Hrsg.): Grundgesetz. Kommentar, 62. Ergänzungslieferung 2011, Art. 20 Rn. 42.

37 RAUE, Frank: Müssen Grundrechtsbeschränkungen wirklich verhältnismäßig sein? Archiv des öffentlichen Rechts, 131 (2006), 79 ff., 108 mit Fn. 99 f.

38 Vgl. nur EuGH, 15. 7. 1970, Rs. C-41/69, Slg 1970, 661, 703 (Chemiefarma); EuGH, 15. 7. 1970, C-44/69, Slg 1970, 733, 763 (Buchler).

39 Vgl. EuGH 7. 6. 1983, Rs. 103-80, Slg 1983, 1825 ff. (MDF Pioneer).

40 BIERMANN: In: IMMENGA - MESTMÄCKER (Hrsg.): Wettbewerbsrecht. Bd. 1, Vor Art. 23 VO 1/2003 Rn. 144 f. 
Art. 15 VO 17/62 entwickelte die Kommission, in Anlehnung an das US-amerikanische Sanktionssystem und die Praxis der US-amerikanischen Behörden, ein System extrem hoher Geldbußen ${ }^{41}$, auch um die Gewinne abzuschöpfen, und verhängt inzwischen sogar Geldbußen im Milliardenbereich.

\section{b. Dezentralisierung der Kartellrechtsdurchsetzung durch die VO 1/2003}

Als die Europäische Union im Jahre 2003 die Verordnung 17/62 durch die neue Verordnung 1/2003 ersetzte ${ }^{42}$, verfolgte sie das Ziel, eine Dezentralisierung der Rechtsanwendung auf dem Gebiet des Kartellrechts einschließlich des kartellrechtlichen Sanktionsrechts zu erreichen. ${ }^{43}$ Die Notwendigkeit der Dezentralisierung war angesichts der Osterweiterung der Europäischen Union erforderlich geworden. Deshalb verzichtete man bewusst darauf, die Mitgliedstaaten zu einer Harmonisierung der Kartellsanktionen zu verpflichten, und hielt es für ausreichend, den Mitgliedstaaten die Pflicht aufzuerlegen, das eigene nationale Sanktionssystem auch auf Rechtsverstöße gegen das EG-Kartellrecht auszuweiten. ${ }^{44}$

Dieser Verzicht der Europäischen Union auf eine Harmonisierung des Kartellsanktionenrechts hatte zur Folge, dass die nationalen Sanktionssysteme der Mitgliedstaaten weiterhin erheblich voneinander abwichen. Die Unterschiede betrafen sowohl die Voraussetzungen, unter denen Verbände und natürliche Personen zur Verantwortung gezogen werden konnten, als auch die angedrohten Rechtsfolgen und die Straf- bzw. Bußgeldbemessung: Die Sanktionsvorschriften reichten von Kriminalstrafen gegen natürliche und juristische Personen (so in England) über Kriminalstrafen gegen natürliche Personen und verwaltungsstraf- oder ordnungswidrigkeitenrechtliche Sanktionen gegen juristische Personen bis zu verwaltungsstrafrechtlichen Sanktionen gegen natürliche und juristische Personen bzw. reinen Verwaltungssanktionen in Griechenland. Diese Rechtslage erwies sich in der Folgezeit als unbefriedigend, da gerade der Schutz des Wettbewerbs innerhalb der Europäischen Union ein zentrales Anliegen in der Europäischen Union war, das eine stärkere Harmonisierung nahe legte. Insbesondere die Problematik der Umgehung von Bußgeldern durch Auflösung oder Veräußerung einer juristischen Person führte zu der Problematik, dass durch Rechtsnachfolgegestaltungen die Sanktionierung in den Mitgliedstaaten ins Leere laufen konnte, während auf EU-Ebene mit dem Unternehmensbußgeldrecht Fälle der Rechtsnachfolge erfasst werden konnten, ohne dass Sanktionslücken auftraten.

41 Näher zu dieser Entwicklung BIERMANN: In: IMMENGA - MESTMÄCKER (Hrsg.): Wettbewerbsrecht. Bd. 1, Vor Art. 23 VO 1/2003 Rn. 92 ff.

42 ABl. vom 4. 1. 2003, L 1/1.

43 DANNECKER, Gerhard: Die Sanktionierung von Verstößen gegen das gemeinschaftsrechtliche Kartellrecht nach der VO (EG) Nr. 1/2003, wistra, 2004, $361 \mathrm{ff}$.

44 Näher dazu DANNECKER, Gerhard: Die Neuregelung der Sanktionierung von Verstößen gegen das EG-Kartellrecht nach der Verordnung (EG) Nr. 1/2003 des Rates vom 16. Dezember 2002 zur Durchführung der in den Art. 81 und 82 des Vertrages niedergelegten Wettbewerbsregeln. In: Festschrift für Ulrich Immenga zum 70. Geburtstag. München, 2004, $61 \mathrm{ff}$. 


\section{c. Harmonisierung des Kartellsanktionsrechts der Mitgliedstaaten unter dem Einfluss der Europäischen Union}

Der europäische Gesetzgeber hat am 11. 12. 2018 die bis zum 4. Februar 2021 umzusetzende Richtlinie (EU) 1/2019 (sog. ECN+-Richtlinie ${ }^{45}$ ) erlassen. Mit Art. 1 Abs. 1 dieser Richtlinie sollte erreicht werden, ,dass die nationalen Wettbewerbsbehörden über die Garantien im Hinblick auf die Unabhängigkeit, über die Ressourcen und die Befugnisse im Bereich der Durchsetzung der Wettbewerbsvorschriften und der Verhängung von Geldbußen verfügen, die sie benötigen, um die Artikel 101 und 102 AEUV wirksam anzuwenden, sodass der Wettbewerb im Binnenmarkt nicht verfälscht wird und den Verbrauchern und Unternehmen keine Nachteile entstehen durch nationale Gesetze und Maßnahmen, die die wirksame Durchsetzung der Wettbewerbsvorschriften durch die nationalen Wettbewerbsbehörden verhindern“. ${ }^{46}$

Zur Erreichung dieser Ziele enthält die ECN+-Richtlinie unter anderem in Erwägungsgrund 46 die Vorgabe, dass der Begriff „Unternehmen“ i.S.d. Art. 101 und 102 AEUV, der im Einklang mit der Rechtsprechung des Gerichtshofs der Europäischen Union anzuwenden ist, als eine wirtschaftliche Einheit zu verstehen ist, auch wenn es sich um mehrere juristische oder natürliche Personen handelt. ${ }^{47}$ Nach Art. 13 Abs. 5 der Richtlinie haben die Mitgliedstaaten sicherzustellen, dass für die Zwecke der Verhängung von Geldbußen gegen Muttergesellschaften sowie rechtliche und wirtschaftliche Nachfolger von Unternehmen der unionsrechtliche Begriff des Unternehmens angewandt wird. ${ }^{48}$ Diesbezüglich besteht in den Mitgliedstaaten die Notwendigkeit, ihr Sanktionssystem unter Verzicht auf das Rechtsträgerprinzip zu ersetzen oder jedenfalls so zu erweitern, dass dieselben Ergebnisse erzielt werden wie bei den Sanktionen im Unionskartellrecht.

45 RL 2019/1/EU des Europäischen Parlaments und des Rates vom 11. 12. 2018 zur Stärkung der Wettbewerbsbehörden der Mitgliedstaaten im Hinblick auf eine wirksamere Durchsetzung der Wettbewerbsvorschriften und zur Gewährleistung des reibungslosen Funktionierens des Binnenmarkts, ABl. L11/3 (14. 1. 2019), dazu ACHENBACH, Hans: Die ECN+-Richtlinie (EU) 2019/1 und das deutsche Kartellordnungswidrigkeitenrecht. wistra, 2019, $257 \mathrm{ff}$.

46 RL 2019/1/EU des Europäischen Parlaments und des Rates vom 11. 12. 2018 zur Stärkung der Wettbewerbsbehörden der Mitgliedstaaten im Hinblick auf eine wirksamere Durchsetzung der Wettbewerbsvorschriften und zur Gewährleistung des reibungslosen Funktionierens des Binnenmarkts, ABl. L11/3 (14. 1. 2019).

47 RL 2019/1/EU des Europäischen Parlaments und des Rates vom 11. 12. 2018 zur Stärkung der Wettbewerbsbehörden der Mitgliedstaaten im Hinblick auf eine wirksamere Durchsetzung der Wettbewerbsvorschriften und zur Gewährleistung des reibungslosen Funktionierens des Binnenmarkts, ABl. L11/3 (14. 1. 2019).

48 Siehe auch ACHENBACH: wistra, 2019, 257, 259. 
In Deutschland wurden diese Vorgaben zu einem einheitlichen Modell der kartellrechtlichen Unternehmensverantwortung, unter Beibehaltung des Rechtsträgerprinzips, verbunden. ${ }^{49}$ Damit ist ein eigenständiges bußgeldrechtliches „Sonderhaftungsrechtsregime“" entstanden, das dem EU-Recht angenähert, mit diesem aber nicht identisch ist. Der wesentliche Unterschied zwischen dem deutschen und dem europäischen Modell liegt darin, dass das deutsche Modell nicht vollständig auf den funktionalen Unternehmensbegriff des europäischen Kartellordnungswidrigkeitenrechts verweist, sondern weiterhin die Verbandsverantwortlichkeit des $\S 30$ OWG, die auf dem Rechtsträgerprinzip beruht, in Bezug nimmt. Sanktioniert werden können nur juristische Personen und sonstige Verbände. Dies hat zur Folge, dass im deutschen Sanktionenrecht auch weiterhin an die Individualtat einer natürlichen Leitungsperson angeknüpft und die wirtschaftliche Einheit lediglich zwischen Mutterund Tochtergesellschaft bei einem entsprechenden Abhängigkeitsverhältnis angenommen wird, während Schwestergesellschaften - anders als im europäischen Recht kein Teil des Unternehmens sind, der in die Ahndung einbezogen werden kann. Außerdem können Muttergesellschaften eigenständig - und nicht als Teil des Unternehmens - geahndet werden, auch wenn ihnen kein schuldhaftes Verhalten vorgeworfen werden kann. Auf diese Weise soll im Ergebnis das Unternehmen sanktioniert werden.

Beachtenswert - sowohl im europäischen als auch im nationalen Kontext - ist weiterhin die Änderung der Richtlinie in Bezug auf den Höchstbetrag der Geldbuße: Nach Art. 14 Abs. 1 der Richtlinie soll der Höchstbetrag nunmehr ,mindestens“ $10 \%$ des weltweiten Gesamtumsatzes im vorausgegangenen Geschäftsjahr betragen, so dass sowohl das bisherige Verständnis der 10\%-Grenze als Kappungsgrenze als auch als Bußgeldobergrenze hinfällig zu sein scheinen. ${ }^{50}$

Praktische Bedeutung haben zudem die Regelungen zur Amtshilfe und zum gerichtlichen Bußgeldverfahren, und zwar nicht zuletzt deshalb, weil Art. 30 der Richtlinie eine Rollenverschiebung festschreibt bzw. mit sich bringt, weil die Kartellbehörden auch weiterhin Verfolgungsbehörden sein müssen. ${ }^{51}$ Damit wird erreicht, dass das Europäische Kartellbußgeldrecht die nationalen Kartellsanktionssystem maßgeblich prägen und die Tendenz der nationalen Kartellbehörden, sich am Unionsrecht zu orientieren, weiter verstärken wird.

49 Eingehend dazu DANNECKER, Gerhard: Das Kartellordnungswidrigkeitenrecht im Wandel: Von der Verbands- zur Unternehmensverantwortung? In: Festschrift für Rudolf Rengier zum 70. Geburtstag. München, 2018, 193 ff.; DANNECKER, Gerhard - MüLLER, Nadja: Das Kartellsanktionenrecht im Lichte der 9. GWB-Novelle. KSzW, 2017, 125, 127 ff.; siehe auch DANNECKER, Gerhard - DANNECKER, Christoph - MÜLLER, Nadja: Das Kartellordnungswidrigkeitenrecht nach der 8. GWB-Novelle: weiterer Reformbedarf? ZWeR, 2013, $417 \mathrm{ff}$.

50 ACHENBACH: wistra, 2019, 257, 259.

51 OsT, Konrad: Die Richtlinie 1/2019: Ein Meilenstein für die Rechtsdurchsetzung im European Competition Network. NZKart, 2019, 69, 70. 


\section{AUSWEITUNG DES UNIONSRECHTLICHEN KARTELLBUBGELDRECHTS GEGEN UNTERNEHMEN AUF DAS SANKTIONSREGIME IM BEREICH DES DATENSCHUTZES UND DER BANKENAUFSICHT}

Die Europäische Union hat das Bußgeldrecht gegen Unternehmen für zwei weitere Bereiche eingeführt: das Datenschutzrecht und das Recht der Bankenaufsicht.

\subsection{Bußgeldregelungen in der Datenschutzgrundverordnung in Anlehnung an das Sanktionsmodell des Europäischen Kartellrechts}

Das Kartellsanktionsrecht der Europäischen Union war Vorbild für eine Bußgeldregelung gegen Unternehmen, die sich in der unionsrechtlichen Datenschutzgrundverordnung findet. Dort droht Art. 83 DSGVO in den Absätzen 4 bis 6 für nahezu jeden Verstoß gegen Verbotsvorschriften der DSGVO Geldbußen an. Dabei kann zwischen „formellen“ und „materiellen“ Verstößen unterschieden werden. ${ }^{52}$ Die Höhe kann - je nach Art des Verstoßes - bis zu 10 Mio. Euro, bei Unternehmen bis zu 2\% des weltweiten Jahresumsatzes, oder aber bis zu 20 Mio. Euro, bei Unternehmen bis zu 4\% des weltweiten Jahresumsatzes, betragen. Nach Art. 83 Abs. 1 DSGVO müssen die Sanktionen wirksam, verhältnismäßig und abschreckend sein. Art. 83 Abs. 2 DSGVO nennt sodann Kriterien für die Bußgeldbemessung und regelt das Verhältnis zu den weiteren Befugnissen der Aufsichtsbehörden. Art. 83 Abs. 3 DSGVO regelt Fälle von Mehrfachverstößen.

\section{a. Das Konzept der Unternehmensverantwortung des Art. 83 DSGVO}

Art. 83 DSGVO sieht in Anlehnung an das Sanktionsmodell des Europäischen Kartellrecht $s^{53}$ die bußgeldrechtliche Verantwortung von Unternehmen vor. ${ }^{54}$ Hiernach können die Handlungen sämtlicher Mitarbeiter des Unternehmens diesem zugerechnet werden. ${ }^{55}$ Welcher Mitarbeiter konkret gehandelt hat ist ohne Bedeutung und muss deshalb nicht nachgewiesen werden. ${ }^{56}$ Grundgedanke der Unternehmensverantwortung ist, dass jedes Unternehmen verpflichtet ist, sich so zu organisieren, dass keine Rechtsverstöße vorkommen. Jedes Unternehmen muss das Tun seiner Mitarbeiter kennen und kann sich nicht darauf berufen, dass seine interne Organisation

52 HOLLÄNDER, Gerrit: In: WOLFF, Heinrich Amadeus - BRINK, Stefan: BeckOK Datenschutzrecht. 33. Ed. 01. 08. 2020, Art. 83 DSGVO Rn. 52; BEHR, Nicolai - TANNEN, Florian: Droht das Zeitalter der Datenschutzgeldbußen? ... und was Unternehmen dagegen tun können. CCZ, 2020, 120, 122.

53 Vgl. AlbRECHT, Jan Philipp: Das neue EU-Datenschutzrecht - von der Richtlinie zur Verordnung. $C R, 2016,88,96$.

54 Vgl. ALBRECHT: $C R, 2016,88,96$.

55 FAUST, Sebastian - SPITTKA, Jan - WYBITUL, Tim: Milliardenbußgelder nach der DSGVO? ZD, 2016, 120, 121; SCHÖNEFELD, Jana - THOMÉ, Sarah: Auswirkungen der Datenschutz-Grundverordnung PinG, 2017, 126.

56 EuGH, 18. 9. 2003, Rs. C-338/00 P, Slg 2003, I-9189, Rn. 98 (Volkswagen). 
nicht fehlerfrei funktioniert hat. ${ }^{57}$ Das Unternehmen wird insbesondere nicht dadurch entlastet, dass sich die Mitarbeiter nicht an die unternehmensintern aufgestellten Regeln gehalten haben. ${ }^{58}$

Die Übernahme des unionsrechtlichen Kartellsanktionsmodells in das Datenschutzrecht bedeutet, dass Bußgeldadressat das Unternehmen ist und die Konzernmuttergesellschaft und die von ihr abhängigen Gesellschaften für Verstöße der Letzteren und die hierfür verhängte Geldbuße haften. Allerdings haftet die Muttergesellschaft nicht für die Unternehmensgeldbuße, wenn sie (ausnahmsweise) nachweisen kann, dass sie keinen bestimmenden Einfluss auf das Verhalten der letztlich den Verstoß begehenden Tochtergesellschaft ausgeübt hat. ${ }^{59}$

Nach Erwägungsgrund 150 S. 3 DSGVO soll der kartellrechtliche Unternehmensbegriff im Sinne von Art. 101 und 102 AEUV auch im Datenschutzrecht Anwendung finden, wenn Geldbußen gegen Unternehmen verhängt werden (siehe Rn. 322). ${ }^{60}$ Allerdings ist umstritten, ob im europäischen Datenschutzrecht der funktionale Unternehmensbegriff des Kartellrechts oder aber ein datenschutzrechtlicher Unternehmensbegriff zugrunde zu legen ist. So vertreten Cornelius und Krohm die Auffassung, der Begriff des Unternehmens in Art. 83 DSGVO sei nicht als wirtschaftliche, sondern als „datenschutzrechtliche Einheit“ zu verstehen: ${ }^{61}$ Von einem Unternehmen sei auszugehen, wenn keine Entscheidungsautonomie innerhalb des Verbundes über das „Ob“ und „Wie“ der Datenverarbeitung besteht. Gestützt wird diese Auffassung darauf, dass die Tatbestände des Kartellrechts und der DSGVO keine strukturelle Ähnlichkeit aufwiesen, denn das Kartellrecht sei durch den Gleichlauf der Adressateneigenschaft des Unternehmens - sowohl Art. 101 und 102 AEUV als auch die Sanktionsnorm des Art. 23 VO (EG) Nr. 1/2003 richten sich an Unternehmen - geprägt. In der DSGVO seien hingegen als Adressaten der Verbote in Art. 5 Abs. 2 DSGVO „die Verantwortlichen“ genannt und in Art. 83 DSGVO Unternehmen als Bußgeldadressaten; Verbots- und Sanktionsadressaten seien also nicht identisch. ${ }^{62}$ Zudem spreche gegen eine Übernahme des kartellrechtlichen Unternehmensbegriffs, dass dieser im unmittelbaren Zusammenhang mit dem Konzernprivileg stehe, das unternehmensinterne Maßnahmen vom Kartellverbot ausnimmt und unternehmensinterne Umstrukturierungen nicht der Fusionskontrolle unterfallen lässt. Dieses Privileg gilt im Datenschutzrecht unstreitig nicht. ${ }^{63}$

57 EuG, 30. 9. 2009, Rs. T-161/05, Slg 2009, II-3555, Rn. 55 (Hoechst).

58 Vgl. EuGH, 18. 7. 2013, Rs. C-501/11 P, EU:C:2013:522 (Schindler Holding).

59 EuGH, 10. 9. 2009, Rs. C-97/08 P, Slg 2009, I-08237, Rn. 60 (Akzo Nobel).

60 Eingehend zur Genese UebelE, Fabian: Das „Unternehmen“ im europäischen Datenschutzrecht. $E u Z W, 2018,440,445$.

61 CoRNELIUS, Kai: Die „datenschutzrechtliche Einheit“ als Grundlage des bußgeldrechtlichen Unternehmensbegriffs nach der EU-DSGVO. NZWiSt, 2016, 421, 424 ff.; КROHM, Niclas: Die wirtschaftliche Einheit als Bußgeldadressat unter der Datenschutz-Grundverordnung? RDV, 2017, 221, 225.

62 CORNELIUS: NZWiSt, 2016, 421, 424 f.; DANNECKER - DANNECKER: NZWiSt, 2016, 162, 168.

63 CoRnelius: NZWiSt, 2016, 421, $424 \mathrm{f}$. 
Überwiegend wird jedoch für das Bußgeldrecht nach der DSGVO die Übernahme des kartellrechtlichen Unternehmensbegriffs befürwortet, da das Konzept einer „datenschutzrechtlichen Einheit" keinen Anknüpfungspunkt in der DSGVO finde. ${ }^{64}$ Hinzu komme, dass hierdurch die wirtschaftliche Realität in Konzernen nicht abgebildet werden könne. Denn eine Muttergesellschaft profitiere von der Datenverarbeitung durch die Tochtergesellschaft unabhängig davon, ob sie die Entscheidungsautonomie über die Datenverarbeitung hat. Dann sei es aber auch nicht sachgerecht, die Frage der Entscheidungsautonomie über die Datenverarbeitung zum Kriterium für die Haftung der Muttergesellschaft zu erklären. Durch das Modell einer „datenschutzrechtlichen Einheit" würden zudem die Zwecke, an denen die Rechtsprechung die Entwicklung des kartellrechtlichen Unternehmensbegriffs ausgerichtet hat, unterlaufen, nämlich zu verhindern, dass die Sanktionsnormen, etwa durch Einschaltung einer unterkapitalisierten Tochtergesellschaft, unterlaufen werden. Diese Gefahr bestünde aber weiterhin, fragte man einzig nach den Entscheidungsbefugnissen hinsichtlich der Datenverarbeitung. ${ }^{65}$ Schließlich spiegele Erwägungsgrund 150 S. 3 der DSGVO den erklärten Willen des Verordnungsgebers wider, den kartellrechtlichen Unternehmensbegriff zur Anwendung zu bringen. Dies belege auch die Genese $^{66}$ dieses Hinweises, die zeige, dass bewusst neben den in Art. 4 Nr. 18 DSGVO legaldefinierten engen Unternehmensbegriff ein weiterer gestellt werden sollte. ${ }^{67}$

Auch wenn Erwägungsgründe, im Gegensatz zum verfügenden Teil der Rechtsakte der Union, als solche nicht verbindlich sind, sind sie doch wichtige Auslegungshilfen. ${ }^{68}$ Dem Verordnungsgeber ist es nicht allein darum gegangen, durch den Verweis in S. 3 des Erwägungsgrundes 150 eine Abgrenzung wirtschaftlicher von nicht-wirtschaftlicher Tätigkeit anzuordnen. ${ }^{69}$ Die Erforderlichkeit dieser $\mathrm{Ab}$ grenzung ergibt sich bereits aus der Definition des Art. 4 Nr. 18 DSGVO, die gerade die Ausübung einer wirtschaftlichen Tätigkeit verlangt. Die Auslegung des Unternehmensbegriffs in Art. 83 DSGVO im Sinne der wirtschaftlichen Einheit wird schließlich durch das in Art. 83 Abs. 1 DSGVO normierte übergeordnete Ziel bedingt, dass die Geldbußen wirksam, verhältnismäßig und abschreckend sein müssen. Ohne die Haftung der Muttergesellschaft wäre dieser jedoch ein weitgehend sanktionsfreier Umgang mit dem Datenschutz möglich, obwohl sie das Verhalten ihrer Tochtergesellschaften steuern kann. ${ }^{70}$ Der funktionale Unternehmensbegriff ermöglicht schließlich, die Fälle der Rechtsnachfolge zu erfassen und nachträgliche gesellschaftsrechtliche Gestaltungen, die zu einer Ahndungslücke führen können, zu

64 Eingehend dazu UEBELE: $E u Z W, 2018,440,443$ ff.

65 UEBELE: EuZW, 2018, 440, 444.

66 Eingehend dazu UEBELE: EuZW, 2018, 440, 445.

67 Zum Gesetzgebungsverfahren allgemein ALBRECHT, Jan Philipp - JoTzO, Florian: Das neue Datenschutzrecht der EU. 2017, Teil 1 Rn. 11-17.

68 EuGH, 19. 11. 2009, Rs. C-407/07, Slg 2009, I-10923 = BeckRS 2010, 90215 Rn. 42 (Sturgeon).

69 So aber FAUST - SPITTKA - WYBITUL: ZD, 2016, 120, 124.

70 Nemitz, Paul: In: EHMANN, Eugen - SELMAYR, Martin: Datenschutz-Grundverordnung: DS-GVO. München, 2. Aufl., 2018, Art. 83 Rn. 43. 
vermeiden. Versteht man „Unternehmen“ in Art. 83 Abs. 4 bis 6 DSGVO im Sinne einer wirtschaftlichen Einheit, so hat das zur Folge, dass neben der Muttergesellschaft jede der Untereinheiten bebußt werden kann, die an dem Verstoß beteiligt war. Der gesamte Konzernumsatz steckt den Bußgeldrahmen ab.

Dieser Auffassung hat sich das Landgericht Bonn ${ }^{71}$ in seinem Urteil vom 11. 11. 2020 - 29 OWi 1/20 angeschlossen, das darlegt, dass für die Ordnungswidrigkeitentatbestände in Art. 83 Abs. 4 bis 6 DSGVO die Grundsätze des supranationalen Kartellrechts entsprechend anzuwenden sind und die Vorschrift des $\S 30$ Abs. $1 \mathrm{OWiG}$ und das deutsche Rechtsträgerprinzip keine Anwendung finden.

Demgegenüber bejahte das Landgericht Berlin in seinem Beschluss vom 18. 02. 2021 die Anwendbarkeit von $\S 30 \mathrm{OWiG}$ und damit das Erfordernis eines schuldhaften Fehlverhaltens einer Führungskraft. ${ }^{72}$ Ein expliziter Ausschluss der Verweisung auf $\S 30$ OWiG im Rahmen von Datenschutzverstößen sei entgegen der ersten Fassung des Referentenentwurfs des BDSG gerade nicht in das aktuell geltende BDSG mit aufgenommen worden. Nur eine natürliche Person könne eine Ordnungswidrigkeit vorwerfbar begehen; einer juristischen Person könne hingegen lediglich das Handeln ihrer Organmitglieder oder Repräsentanten zugerechnet werden. In der Folge hob das LG Berlin den Bußgeldbescheid der Aufsichtsbehörde auf.

Während das Urteil des LG Bonn rechtskräftig geworden ist, legte die die Staatsanwaltschaft Berlin Beschwerde ein, so dass sich das Kammergericht Berlin dieser Streitfrage annehmen wird. Die Auffassung des Landgerichts Berlin würde dazu führen, dass der Aufwand zur Sachverhaltsermittlung für die Aufsichtsbehörden deutlich steigen würde und wohl häufig ein konkreter Verstoß einer individualisierten Führungskraft nicht nachweisbar sein wird. ${ }^{73}$

Ebenso wie das Landgericht Berlin entschied das österreichische Bundesverwaltungsgericht zwei kurz aufeinander folgenden Entscheidungen. ${ }^{74}$ Das Gericht sah sich durch das europarechtliche Effektivitätsgebot nicht gehindert, eine unmittelbare Unternehmenshaftung sui generis DS-GVO abzulehnen. Vielmehr hat es unter Hinweis auf die durch Art. 83 Abs. 8 DS-GVO verbürgte Gewährleistung nationaler Verfahrensgarantien eine ,ausreichende Bestimmtheit der natürlichen Person, deren

71 LG Bonn Urt. v. 11. 11. 2020 BeckRS 2020, 35663 Rn. 70; dazu VENN, Nikolai - WYBITUL, Tim: Die bußgeldrechtliche Haftung von Unternehmen nach Art. 83 DS-GVO (zugl. Anm. zu LG Bonn Urt. v. 11. 11. 2020 - 29 OWi 1/20), NStZ 2021, 204 ff.

72 Landgericht Berlin, Beschluss v. 18. 02. 2021 - 526 OWi LG 212 Js-OWi 1/20.

73 Näher zu diesen beiden Urteilen VON WALTER, Axel: EU-Verbandsanktionen durch die DSGVO? LG Bonn und LG Berlin unvereinbar. Datenschutz-Berater, 2021, $98 \mathrm{ff}$.

74 Entscheidung des Bundesverwaltungsgerichts der Republik Österreich v. 19. 8. 2019, Az. W211 2208885-1, https://www.ris.bka.gv.at/Dokumente/Bvwg/BVWGT_20190819_W 211_2217629_1_00/BVWGT_20190819_W211_2217629_1_00.pdf; Erkenntnis des Bundesverwaltungsgerichts der Republik Österreich vom 26. 11. 2020, Az: W258 2227269-1/14E, https://www.ris.bka.gv.at/Dokumente/Bvwg/BVWGT_20201126_W25 8_2227269_1_00/BVWGT_20201126_W258_2227269_1_00.pdf. 
Verhalten der juristischen Person zugerechnet werden soll“, gefordert. ${ }^{75}$ Die Tat müsse entsprechend den Vorgaben des österreichischen Rechts hinsichtlich des Täters und der Tatumstände so genau umschrieben sein, dass die Zuordnung des Tatverhaltens zur Verwaltungsvorschrift, die durch die Tat verletzt worden ist, in Ansehung aller Tatbestandsmerkmale ermöglicht wird. ${ }^{76}$

Letztlich wird der EuGH im Rahmen eines Vorlageverfahrens über die unmittelbare Anwendbarkeit der Datenschutzgrundverordnung und damit über das unionsrechtliche Unternehmensbußgeldrecht entscheiden müssen.

\section{b. Allgemeiner Teil, insbesondere Verschuldenserfordernis}

Die Anerkennung eines Unternehmensbußgeldrechts auf dem Gebiet des Datenschutzrechts hat zur Folge, dass die Vorschriften des Allgemeinen Teils der nationalen Sanktionsrechtsordnungen nicht auf die unionsrechtlichen Geldbußen angewendet werden dürfen ${ }^{77}$, sondern die ungeschriebenen Regeln des Allgemeinen Teils des Kartellordnungswidrigkeitenrechts, das für die bußgeldrechtliche Verantwortung nach Art. 83 DSGVO Pate stand, heranzuziehen sind. ${ }^{78}$ Diesbezüglich stellt sich die Rechtslage in Deutschland bei der Sanktionierung von Rechtsverstößen gegen Art. 101 und 102 AEUV auf der Grundlage des $\S 81$ GWB anders dar, weil hier nicht Bußgeldvorschriften der EU, sondern des nationalen Rechts - § $81 \mathrm{GWB}$ iVm $\S 30$ OWiG - zur Anwendung kommen.

\section{c. Unternehmensbezogener Bußgeldrahmen und verantwortungsbezogene Buß- geldbemessung}

Während der Gesamtjahresumsatz bei Art. 23 VO (EG) Nr. 1/2003 von 10\% des Gesamtjahresumsatzes lediglich als eine Kappungsgrenze verstanden wird, ist für Art. 83 DSGVO von einer Bußgeldobergrenze von bis zu 2 bzw 4\% des Gesamtjahresumsatzes auszugehen. ${ }^{79}$ Dies ergibt sich bereits aus dem Wortlaut der Regelungen: Während es in Art. 23 Abs. 2 UAbs. 2 VO (EG) Nr. 1/2003 heißt, dass „die Geldbuße [...] 10\% [des Gesamtjahresumsatzes] nicht übersteigen" darf, so dass ein Übersteigen der Grenze zwar rechnerisch möglich, an dieser Grenze der Betrag aber zu kappen ist, sieht Art. 83 Abs. 4 bis 6 DSGVO vor, dass Geldbußen „von bis zu 2 Prozent [bzw. 4 Prozent des Gesamtjahresumsatzes] verhängt“ werden. Diese Rege

75 Entscheidung des Bundesverwaltungsgerichts der Republik Österreich v. 19. 8. 2019, Az.: W211 2217629-1.

76 Erkenntnis des Bundesverwaltungsgerichts der Republik Österreich vom 26. 11. 2020, Geschäftszahl: W258 2227269-1/14E.

77 So aber BERGT, Matthias: Datenschutz und Datensicherheit. DuD, 2017, 555, 558.

78 Dazu BAUER, Raphaela: Der Allgemeine Teil des Kartellbußenrechts. Wien 2020, $149 \mathrm{ff}$.

79 UEBELE: EuZW, 2018, 440, 446; a.A. GRÜNWALD, Andreas - HACKL, Jens: Das neue umsatzbezogene Sanktionsregime der DS-GVO. ZD, 2017, 556, 557, $559 \mathrm{f}$. 
lung steckt einen bestimmten Bußgeldrahmen mit fester Obergrenze ab, innerhalb dessen die Geldbuße von vornherein zu bestimmen ist. ${ }^{80}$

Im Rahmen der Bußgeldbemessung ist nach dem Erwägungsgrund 150 zur DSGVO der funktionale Unternehmensbegriff des europäischen Kartellrechts in Art. 101 und 102 AEUV zugrunde zu legen. Daher kommt es bei der Bestimmung der Obergrenze einer möglichen Geldbuße auf den Gesamtumsatz des betroffenen Konzerns als Unternehmen im funktionalen Sinne an. Allerdings kann der Gesamtumsatz allenfalls einen gewissen Bezug zur Leistungsfähigkeit aufweisen. Unrecht und Schuld der Tat spiegeln sich darin in keiner Weise wider.

Art. 83 Abs. 2 DSGVO sieht für die Bemessung der Geldbuße einen Katalog von Zumessungskriterien vor. Als zu berücksichtigende Faktoren werden Art, Schwere und Dauer des Verstoßes, Vorsatz oder Fahrlässigkeit, Wiederholungsfall, Zusammenarbeit mit den Behörden erwähnt. Ferner werden die Ermittlung und Aufklärung der Zuwiderhandlung sowie datenschutzspezifische Faktoren wie die Art der betroffenen (persönlichen) Daten oder die Einhaltung von bestimmten Verhaltensregeln bzw. Zertifizierungsverfahren genannt. Hingegen wird auf den erzielten Umsatz als Bußgeldbemessungskriterium nicht verwiesen.

Das Landgericht Bonn ${ }^{81}$ führt bezüglich der Bußgeldbemessung aus: Die Höhe des Umsatzes sei für die Unternehmensgröße und damit für die Ahndungsempfindlichkeit ein geeigneter Indikator. Es dürfe jedoch nicht aus dem Blick geraten, dass die DSGVO in Art. 83 Abs. 2 S. 2 in erster Linie tatbezogene Gesichtspunkte für die Bemessung aufführt. Eine Bemessung des Bußgeldes durch Ermittlung eines sich nach dem Umsatz richtenden Grundwertes für das Bußgeld, welcher je nach Schwere des Datenschutzverstoßes mit einem Faktor multipliziert wird, sei problematisch. Eine solche Bemessungsmethode versage bei schweren Datenschutzverstößen umsatzschwacher Unternehmen und leichten Datenschutzverstößen umsatzstarker Unternehmen. Die Bemessung innerhalb dieses Rahmens richte sich nach Art. 83 Abs. 1 DSGVO. Das Bußgeld müsse danach wirksam, verhältnismäßig und abschreckend sein.

Das Gericht verweist sodann auf die Kriterien aus Art. 83 Abs. 2 S. 2 DSGVO. Der Umsatz werde darin nicht genannt. Dies bedeute jedoch nicht, dass dieser für die Bemessung keine Bedeutung habe. Er spiele im Rahmen der Ahndungsempfindlichkeit eine Rolle, die von der Größe des Unternehmens abhänge. Dafür sei wiederum der Umsatz des Unternehmens maßgeblich. Zusätzlich könne der Gewinn berücksichtigt werden. Zu beachten sei jedoch, dass der Umsatz nur eines der Kriterien sei. Art. 83 Abs. 2 DSGVO nenne tatbezogene Umstände. Eine Bemessungsformel, ähnlich der Berechnung von Tagessätzen im Strafgesetzbuch, sei dagegen nicht vorgesehen. Eine solche Formel könne lediglich eine erste Annäherung sein. Die Kriterien aus Art. 83 Abs. 2 DSGVO würden gegenüber dem Umsatzkriterium überwiegen, je weiter die Bewertung in die eine oder andere Richtung ausschlage. Bei ge-

80 Uebele: EuZW, 2018, 440, 446; PAAl, Boris - PAUly, Daniel A. - FrenZEL, Eike Michael: Datenschutz-Grundverordnung. 2. Aufl., 2018, Art. 83 Rn. 18.

81 LG Bonn, Urteil vom 11. 11. 2020 - 29 OWi 1/20 = BeckRS 2020, 35663. 
ringen Verstößen umsatzstarker Unternehmen müsse das Kriterium der Verhältnismäßigkeit bedacht werden. Danach müsse das Bußgeld zwar spürbar, aber nicht unangemessen hoch sein. Hier seien sämtliche mildernde Umstände zu berücksichtigen. Im konkreten Fall führte dies zu einer deutlichen Herabsetzung der Geldbuße von 4,9 Mio. Euro auf 900000 Euro.

Die Entscheidung des Landgerichts Bonn, die den Schwerpunkt auf das Unrecht legt und die Verhältnismäßigkeit zum Maßstab wählt - richtiger wäre es gewesen, auf die Schuld abzustellen, denn sowohl für Strafen als auch für Geldbußen ist der Maßstab nicht die Verhältnismäßigkeit, sondern die Schuldangemessenheit der Sanktion - vermeidet die Problematik, dass der Umsatz ohne hinreichende Aussagekraft für die Leistungsfähigkeit ist. Wenn ein Unternehmen ein Produkt veräußert, ist der Umsatz deutlich höher, als wenn es nur eine Lizenz für die Herstellung eines Produkts, das vom Produzenten direkt an den Abnehmer veräußert wird, erhebt. Am Gewinn des Unternehmens und seiner Leistungsfähigkeit muss sich trotz des unterschiedlichen Umsatzes nichts ändern. Daher ist es richtig, auf das Unrecht und die Schuld der Tat abzustellen. Dadurch relativieren sich die zunächst gravierend erscheinenden Unterschiede zwischen der Sanktionierung des Rechtsträgers und des Unternehmens.

Allerdings trägt das von der Konferenz der unabhängigen Datenschutzaufsichtsbehörden des Bundes und der Länder („DSK“) am 14. 10. 2019 veröffentlichte Konzept zur Bemessung der Geldbußen gem. Art. 83 DSGVO (,Konzept zur Bußgeldbemessung" $)^{82}$ diesen Anforderungen nicht Rechnung. Nach diesem Konzept soll die Ermittlung einer Geldbuße wie folgt erfolgen: ${ }^{83}$ Zunächst wird das betroffene Unternehmen einer Größenklasse zugeordnet (1), sodann der mittlere Jahresumsatz der jeweiligen Untergruppe der Größenklasse bestimmt (2) und ein wirtschaftlicher Grundwert ermittelt (3); dieser Grundwert wird mittels eines schwereabhängigen Faktors multipliziert (4) und abschließend der so ermittelte Wert anhand täterbezogener und sonstiger noch zu berücksichtigender Umstände angepasst (5). Dieses vorläufige Konzept verliert seine Gültigkeit, sobald der Europäische Datenschutzausschuss (EDSA) seine abschließenden Leitlinien zur Methodik der Festsetzung von Geldbußen erlassen hat.

\section{d. Opportunitätsprinzip}

Nach $\S 47 \mathrm{OWiG}$ gilt im deutschen Ordnungswidrigkeitenrecht das Opportunitätsprinzip, das die Verfolgung einer Ordnungswidrigkeit in das Ermessen der Behörde stellt; auch das Gericht kann das Verfahren einstellen. § 41 Abs. 2 BDSG n.F. erklärt das OWiG nahezu vollständig für anwendbar, insbesondere auch das Opportunitätsprinzip. Art. 83 Abs. 2 S. 1 DSGVO sieht jedoch vor, dass bei Verstößen eine Ahndung mittels Geldbuße verpflichtend ist und Maßnahmen zur Abstellung des Verstoßes nach

82 https://www.datenschutzkonferenz-online.de/media/ah/20191016_bu\%C3\%9Fgeldkonzept.pdf (Konzept zur Bußgeldbemessung); zuletzt abgerufen am 26. 6. 2020.

83 Siehe dazu BEHR, Nicolai - TANNEN, Florian: Droht das Zeitalter der Datenschutzgeldbußen? ... und was Unternehmen dagegen tun können. CCZ, 2020, 120, $122 \mathrm{ff}$. 
Art. 58 Abs. 2 DSGVO vorgehen. Ein Absehen von einer Geldbuße kommt nur in Betracht, wenn die Bebußung einer natürlichen Person in Frage steht, die für diese eine unverhältnismäßige Belastung darstellen würde, oder wenn der Verstoß geringfügig ist, was letztlich dem unionsrechtlichen Verhältnismäßigkeitsgrundsatz entspricht. ${ }^{84}$ Insoweit hat das EU-Recht Vorrang vor dem nationalen Recht.

\section{e. Verjährung}

Im Gesetzgebungsprozess wurde die Verjährung auf EU-Ebene nicht geregelt. Diesbezüglich soll es möglich sein, hilfsweise auf das nationale Recht zurückzugreifen, ${ }^{85}$ solange dieses die abschreckende Wirkung des Art. 83 DSGVO nicht gefährde. ${ }^{86}$ Dabei habe man sich an den unionsrechtlichen Regelungen der VO 2988/74 und der Art. 25, 26 KartellverfahrensVO zu orientieren. ${ }^{87}$ Dies widerspricht jedoch der Rechtsprechung des EuGH, der in Bezug auf die Kartellordnungswidrigkeiten dargelegt hat, dass es sich bei der Verjährung um einen allgemeinen Rechtsgrundsatz handelt, der jedoch einer gesetzlichen Regelung bedürfe.

\subsection{Durch die Europäische Zentralbank und (subsidiär) durch nationale Zent- ralbanken zu verhängende unionsrechtliche Geldbußen gegen juristische Personen und gegen Unternehmen}

Die Kompetenzen der Europäischen Union zur Festsetzung von Geldbußen gegen Unternehmen umfassen gemäß Art. 132 Abs. 3 AEUV auch die Verhängung von Sanktionen im Rahmen der Bankenaufsicht. Zuständig hierfür sind die Europäische Zentralbank bzw. die nationalen Zentralbanken der Mitgliedstaaten.

\section{a. Verordnung (EG) Nr. 2532/98 des Rates vom 23.11.1998 über das Recht der Europäischen Zentralbank, Sanktionen zu verhängen}

Auf der Grundlage des Art. 132 Abs. 3 AEUV wurde die „Verordnung (EG) Nr. 2532/98 des Rates vom 23.11.1998 über das Recht der Europäischen Zentralbank, Sanktionen zu verhängen “88, erlassen. Darin sind Unternehmensgeldbußen vorgese-

84 SChreIBAUER, Marcus - SPITTKA, Jan: In: WyBITUL, Tim: EU-Datenschutz-Grundverordnung. 2017, Art. 83 Rn. 13; BERGT, Matthias: In: KÜHLING, Jürgen - BUCHNER, Benedikt: Datenschutz-Grundverordnung, Bundesdatenschutzgesetz: DS-GVO/BDSG. 3. Aufl., 2020, Art. 83 Rn. 32.

85 Becker, Thomas: In: Plath, Kai Uwe (Hrsg.): DSGVO/BDSG. 3. Aufl., 2018, Art. 83 Rn. 3; BERGT: In: KÜHLING - BUCHNER: Datenschutz-Grundverordnung, Bundesdatenschutzgesetz: DS-GVO/BDSG. Art. 83 Rn. 113; kritisch HOLLÄNDER, Gerrit: In: BeckOK, DatenschutzR. 33. Ed. 01. 08. 2020, Art. 83 DSGVO Rn. 29.

86 So BERGT: DuD, 9/2017, 555, 560.

87 BERGT: In: KÜHLING-BUCHNER: Datenschutz-Grundverordnung, Bundesdatenschutzgesetz: $D S-G V O / B D S G$. Art. 83 Rn. 11.

88 VO (EG) 2532 vom 23. 11. 1998, ABl. EU L 318 vom 27. 11. 1998, S. 4 ff. 
hen, wenn die sich aus den Verordnungen und Beschlüssen der europäischen Zentralbank ergebenden Verpflichtungen nicht eingehalten werden. Zur Sicherstellung der Anwendung der Aufsichtsregeln und - beschlüsse sollen wirksame, verhältnismäßige und abschreckende Sanktionen verhängt werden. Diese Verordnung enthält neben Begriffsbestimmungen, unter anderem zu Unternehmen, die Regelung der Verfahrensinitiative, die bei dem EZB-Direktorium und den nationalen Zentralbanken liegt, sowie die Obergrenzen für Geldbußen und Strafgelder und die grundlegenden Verfahrensregeln.

Nach Art. 1 Ziff. 3 sind Unternehmen „,natürliche oder juristische Personen des privaten oder öffentlichen Rechts innerhalb eines teilnehmenden Mitgliedstaats, (...) die Verpflichtungen unterliegen, die sich aus Verpflichtungen und Entscheidungen der EZB ergeben, einschließlich der Zweigstellen oder sonstigen ständigen Niederlassungen, die Unternehmen, die ihre Hauptverwaltung oder ihren juristischen Sitz außerhalb eines teilnehmenden Mitgliedstaats haben, in einem teilnehmenden Mitgliedstaat unterhalten“. Die Obergrenze der Geldbußen liegt nach Art. 2 Abs. 1 lit. a) der Verordnung bei 500000 Euro.

\section{b. VO (EG) Nr. 2157/1999 der Europäischen Zentralbank vom 23. 9.1999 über das Recht der Europäischen Zentralbank, Sanktionen zu verhängen}

Zur Ausführung und Konkretisierung der Rats-VO (EG) Nr. 2532/98 hat der EZBRat dann die VO (EG) Nr. 2157/1999 der Europäischen Zentralbank vom 23. 9.1999 über das Recht der Europäischen Zentralbank, Sanktionen zu verhängen, erlassen. ${ }^{89}$

\section{c. Verordnung (EU) Nr. 1024/2013 - besondere Aufgaben der Europäischen Zentralbank im Zusammenhang mit der Aufsicht über Kreditinstitute}

Mit der Verordnung (EU) Nr. 1024/2013 - besondere Aufgaben der Europäischen Zentralbank im Zusammenhang mit der Aufsicht über Kreditinstitute ${ }^{90}$ - wurde ein einheitlicher Aufsichtsmechanismus (erste Säule) zur Überwachung von Banken im Euro-Währungsgebiet und in den übrigen teilnehmenden EU-Ländern eingerichtet, bestehend aus der EZB und den nationalen Aufsichtsbehörden. Die EZB überwacht bedeutende Banken unmittelbar. Sie ist unter anderem befugt, aufsichtliche Überprüfungen, Prüfungen vor Ort und Untersuchungen durchzuführen und Verwaltungssanktionen bei Verstößen gegen das EU-Recht über Kreditinstitute, Finanzholdinggesell

89 AB1. 1999 L 264/21, geändert durch VO (EG) Nummer 985/2001, ABL 2001 L 137/24 sowie durch VO (EG) Nr. 469/2014, ABl. 2014 L 143/51.

90 SSM-Verordnung (EU) Nr. 1024/2013 (Einheitlicher Aufsichtsmechanismus) - Verordnung (EU) Nr. 1024/2013 des Rates vom 15. Oktober 2013 zur Übertragung besonderer Aufgaben im Zusammenhang mit der Aufsicht über Kreditinstitute auf die Europäische Zentralbank (Einheitlicher Aufsichtsmechanismus), ABl. (EU) L 287 vom 29. 10. 2013, S. 63; zuletzt geändert durch die Berichtigung der Verordnung (EU) Nr. 1024/2013 vom 19. 08. 2015, AB1. L 218/82). 
schaften und gemischte Finanzholdinggesellschaften auf der Grundlage des Art. 18 dieser Verordnung zu verhängen.

Normadressaten sind Kreditinstitute, Finanzholdinggesellschaften oder Gemischte Finanzholdinggesellschaften, die gegen eine Anforderung aus direkt anwendbaren Rechtsakten der Union verstoßen. Kreditinstitut ist „ein Unternehmen, dessen Tätigkeit darin besteht, Einlagen oder andere rückzahlbare Gelder des Publikums entgegenzunehmen und Kredite für eigene Rechnung zu gewähren“. Eine „FinanzholdingGesellschaft" ist ein Unternehmen, dessen Tochterunternehmen ausschließlich oder hauptsächlich Finanzinstitute oder -unternehmen sind, wobei mindestens eines dieser Tochterunternehmen ein Kreditinstitut oder eine Wertpapierfirma ist. ${ }^{91}$ Mit der Regulierung von Finanzholding-Gesellschaften wird das Ziel verfolgt, Unternehmensgruppen, zu denen Kreditinstitute und Wertpapierunternehmen gehören, die aber nicht das Mutterunternehmen der Unternehmensgruppe sind, zu beaufsichtigen. Eine „Gemischte Finanzholdinggesellschaft" ist ein nicht der Aufsicht unterliegendes Mutterunternehmen, das zusammen mit seinen Tochterunternehmen, von denen mindestens eines ein beaufsichtigtes Unternehmen mit Sitz in der Gemeinschaft ist, und anderen Unternehmen ein Finanzkonglomerat bildet. Diese Unternehmen sind Sanktionsadressaten der unionsrechtlichen Bußgeldvorschriften, die durch die EZB zu verhängen sind.

Bei Verstößen gegen nationale Rechtsvorschriften im Zusammenhang mit dem einheitlichen Aufsichtsmechanismus (SSM) sind die nationalen Behörden weiterhin für die Verhängung der Verwaltungssanktionen zuständig; sie sollten solche Sanktionen aber nur gegen direkt von der EZB beaufsichtigte Kreditinstitute verhängen, wenn die EZB sie dazu auffordert, zu diesem Zweck ein Verfahren einzuleiten.

\section{d. Verordnung (EU) 2017/2094 der Europäischen Zentralbank vom 3. 11. 2017 zur Änderung der Verordnung (EU) Nr. 795/2014 zu den Anforderungen an die Überwachung systemrelevanter Zahlungsverkehrssysteme (EZB/2017/32)}

Als Beispiel einer Sanktionsregelung kann Art. 23 der Verordnung (EU) 2017/2094 der Europäischen Zentralbank vom 3.11.2017 zur Änderung der Verordnung (EU) Nr. 795/2014 zu den Anforderungen an die Überwachung systemrelevanter Zahlungsverkehrssysteme (EZB/2017/32) ABl. L 299/11 vom 16. 11. 2017, genannt werden: Diese Sanktionsregelung lautet: „Im Fall eines Verstoßes gegen diese Verordnung kann die EZB Sanktionen verhängen. Solche Sanktionen ergehen im Einklang mit der Verordnung (EG) Nr. 2532/98 des Rates und der Verordnung (EG) Nr. 2157/99 der Europäischen Zentralbank (EZB/1999/4). Die EZB erlässt einen Beschluss zur Methodik zur Berechnung der Höhe der Sanktionen.“

91 Art. 4 Abs. 1 Nr. 20 der Verordnung (EU) Nr. 575/2013 des Europäischen Parlaments und des Rates vom 26. Juni 2013 über Aufsichtsanforderungen an Kreditinstitute und Wertpapierfirmen und zur Änderung der Verordnung (EU) Nr. 646/2012. 
Die Grundsätze und das Verfahren für die Verhängung von Verwaltungsgeldbußen bei Verstößen gegen unmittelbar anwendbares Recht der Union gemäß Art. 18 Abs. 1 der Verordnung (EU) Nr. 1024/2013 sind in der Verordnung (EU) Nr. 1024/2013 und in der Verordnung (EU) Nr. 468/2014 (EZB/2014/17) der Europäischen Zentralbank $^{92}$ festgelegt.

Gegen den Beschluss über die Auferlegung eines Bußgeldes können vor dem Gerichtshof der Europäischen Union Rechtsmittel eingelegt werden. Dabei sind die in Art. 263 AEUV genannten Bedingungen und Fristen einzuhalten.

Bei Verstößen gegen nationale Rechtsvorschriften im Zusammenhang mit dem einheitlichen Aufsichtsmechanismus (SSM) sind die nationalen Behörden weiterhin für die Verhängung der Verwaltungssanktionen zuständig; sie sollten solche Sanktionen aber nur gegen direkt von der EZB beaufsichtigte Kreditinstitute verhängen, wenn die EZB sie dazu auffordert, zu diesem Zweck ein Verfahren einzuleiten.

\section{AUSSTRAHLUNG DER BUBGELDRECHTLICHEN UNTERNEHMENSVERANT- WORTUNG AUF DEN ENTWURF EINES VERBANDSSANKTIONENGESETZES IN DEUTSCHLAND}

Die Strafbarkeit juristischer Personen ist in Deutschland ein Reizthema, das von begeisterten Anhängern dieser Idee gefordert und von vehementen Gegnern aber heftig abgelehnt wird. ${ }^{93}$ Die große Mehrzahl der EU-Mitgliedstaaten kennt inzwischen eine Verbandsstrafbarkeit. ${ }^{94}$ Es besteht jedoch keine auch nur annähernd einheitliche Gesetzeslage in den Mitgliedstaaten. Jeder Mitgliedstaat bestimmt in eigener Verantwortung, ob und unter welchen Voraussetzungen Kriminalstrafen gegen Verbände verhängt werden können. Diese Entwicklung beruht im Wesentlichen auf Vorgaben der Europäischen Union, die in zahlreichen Rechtsakten die Mitgliedstaaten verpflichtet hat, strafrechtliche Sanktionen - Kriminalstrafen oder Geldbußen - gegen juristische Personen und Personenverbände einzuführen, um sie für Straftaten aus dem Unternehmen heraus oder für Straftaten, die im Interesse des Unternehmens begangen werden, verantwortlich zu machen. Die verhängten Sanktionen müssen wirksam, verhältnismäßig und abschreckend sein. ${ }^{95}$

92 Verordnung (EU) Nr. 468/2014 der Europäischen Zentralbank vom 16. April 2014 zur Einrichtung eines Rahmenwerks für die Zusammenarbeit zwischen der Europäischen Zentralbank und den nationalen zuständigen Behörden und den nationalen benannten Behörden innerhalb des einheitlichen Aufsichtsmechanismus (SSM-Rahmenverordnung) (EZB/2014/17).

93 Zusammenfassend dazu Rogall, Klaus: In: MitsCH, Wolfgang (Hrsg.): Karlsruher Kommentar zum Gesetz über Ordnungswidrigkeiten. 5. Aufl., 2018, § 30 Rn. 9 ff., 13.

94 Siehe dazu den Überblick bei TIEDEMANN, Klaus: Wirtschaftsstrafrecht. München, 5. Aufl., 2017, Rn. 446 ff.

95 Näher dazu DANNECKER, Gerhard: Strafe oder bloße Ordnungswidrigkeit - europäische Vorgaben und Entwicklungen. In: LEITNER, Roman - BRANDL, Rainer (Hrsg.): Finanzstrafrecht 2016. Wien, 2017, $125 \mathrm{ff}$. 


\subsection{Der Entwurf eines Verbandssanktionengesetzes}

In Deutschland steht die Einführung einer strafrechtlichen Verantwortung juristischer Personen und sonstiger Verbände in Anlehnung an das österreichische Verbandsverantwortungsgesetz im Gesetzgebungsverfahren in der Diskussion. Das in dem Gesetzesentwurf ${ }^{96}$ vorgesehene System geht von einer Verantwortlichkeit des Verbandes, nicht des Unternehmens, aus, wenn eine Leitungsperson des Rechtsträgers Verband eine Straftat oder Ordnungswidrigkeit begangen hat. Mutter- und Schwestergesellschaften können nur verantwortlich gemacht werden, wenn sie sich an dem Rechtsverstoß über ihre Leitungspersonen beteiligt haben. Sie können dann eigenständig bestraft werden.

\section{2. Übernahme von Elementen aus dem Unternehmensbußgeldrecht in das Verbandssanktionengesetz}

Trotz Beibehaltung des Rechtsträgerprinzips, finden sich im Verbandssanktionengesetz Elemente, die dem Unternehmensbußgeldrecht entnommen sind:

Obwohl Täter der Verbandstat der Rechtsträger, in der Regel eine juristische Person, ist, bestimmt sich der Strafrahmen nicht nach dem Umsatz der zu bestrafenden juristischen Person, sondern nach dem Umsatz des Unternehmens im Sinne einer wirtschaftlichen Einheit, wenn es sich um eine einem Konzern angehörende juristische Person handelt.

Außerdem ist vorgesehen, dass gegen eine Muttergesellschaft, die auf ihre Tochtergesellschaften beherrschenden Einfluss ausgeübt hat, eine Geldbuße auch dann verhängt werden kann, wenn gegen die Muttergesellschaft kein Schuldvorwurf im Hinblick auf die begangene Straftat gemacht werden kann.

In beiden Bereichen - Bestimmung der Bußgeldhöhe nach dem Umsatz der wirtschaftlichen Einheit und Verantwortung der Einfluss ausübenden Muttergesellschaft, unabhängig vom Vorliegen schuldhaften Verhaltens - spiegelt sich der Einfluss des europäischen Kartellsanktionenrechts wider - ein Rechtsgebiet, das sich ganz eigenständig und losgelöst vom Strafrecht in den Mitgliedstaaten entwickelt hat. Die dadurch entstehenden Spannungen im deutschen Verbandssanktionengesetz - Normadressaten sind hier die Rechtsträger, für den Sanktionsrahmen bestimmend ist der Umsatz der wirtschaftlichen Einheit - ist nur dann mit den strafrechtlichen Grundprinzipien, dem Schuldgrundsatz und der schuldangemessenen Bestrafung zu vereinbaren, wenn der Bußgeldrahmen auch nur als Rahmen verstanden wird, die konkret zu verhängende Strafe aber gleichwohl nach dem Unrechts- und Schuldgehalt der Tat und der Leistungsfähigkeit des Normadressaten bestimmt wird.

96 Entwurf eines Gesetzes zur Stärkung der Integrität in der Wirtschaft, BundestagsDrucks. 19/23568. 


\section{NOTWENDIGKEIT EINER ANGLEICHUNG DER MITGLIEDSTAATLICHEN SANKTIONSSYSTEME AN DAS UNIONSRECHTLICHE BUßGELDRECHTS GEGEN UNTERNEHMEN}

Während die Mitgliedstaaten vorrangig strafrechtliche Sanktionen gegen Individuen vorsehen und nur ergänzend Sanktionen gegen juristische Personen und sonstige Verbände (Rechtsträgerprinzip) kennen, sieht das Bußgeldrecht der Europäischen Union ausschließlich Bußgelder gegen Unternehmen vor. Angesichts der Strafrechtsähnlichkeit der Bußgelder ${ }^{97}$ ist eine Annäherung der tatbestandsmäßigen Voraussetzungen der Unternehmensbußgelder und der Verbandsstrafen wie auch der Höhe der straf- und bußgeldrechtlichen Sanktionen bei gleichem Unrecht und Schwere der Tat und gleicher Leistungsfähigkeit geboten. Der deutsche Gesetzgeber will diesen Schritt mit dem Entwurf des Verbandssanktionengesetz gehen.

\subsection{Vereinheitlichung der Sanktionsbemessung bei Unternehmensgeldbußen und Verbandsstrafen}

Um aber tatsächlich eine weitgehende Harmonisierung auch auf der Rechtsfolgenseite zu erreichen, bedarf es über die Vereinheitlichung des Sanktionsrahmens hinaus der Einführung von Sentencing Guidelines auch im Verbandsstrafrecht; für die Ausgestaltung kann auf die langjährigen Erfahrungen im Kartellrecht zurückgegriffen werden. Dort wurden zum 1. 9. 2006 Bußgeldleitlinien erlassen, die der Fortentwicklung und Verfeinerung der Bußgeldpolitik der Kommission dienten. Der Grundbetrag der Geldbuße richtet sich nach Schwere und Dauer der Zuwiderhandlung. Bezugsgröße ist dabei der Umsatz, der mit der Zuwiderhandlung in einem direkten oder indirekten Zusammenhang steht (tatbezogener Umsatz). Der Grundbetrag hat zwei Bestandteile: einen variablen Betrag, der sich aus der Anwendung eines Prozentsatzes von bis zu 30\% auf den tatbezogenen Umsatz sowie der Multiplikation mit der Dauer der eigenen Tatbeteiligung errechnet, sowie einem davon unabhängigen Betrag von bis zu 25\% des tatbezogenen Umsatzes, der auch bei ganz kurzen Zuwiderhandlungen zur Anwendung kommt und als sog. Eintrittsgebühr bezeichnet wird (Z 7). Bezüglich der Dauer wird ein Faktor in Höhe von 100\% pro Jahr angenommen, wobei jedoch zeitliche Abstufungen vorgenommen werden. In einem zweiten Schritt wird der so ermittelte Grundbetrag bei Vorliegen erschwerender oder mildernder Umstände angepasst. ${ }^{98}$ Allerdings ist hierbei die 10\%-Obergrenze nach Art. 23 Abs. 2 VO 1/2003 zu beachten, nach der eine zu verhängende Geldbuße gekappt werden kann. ${ }^{99}$ Hingegen haben sich die höchstrichterliche Rechtsprechung in Deutschland und Österreich dafür ausgesprochen, die 10\%-Grenze als Sanktionsobergrenze zu verstehen und anzuwenden.

97 So HENN: a.a.O. 32.

98 BIERMANN: In: IMMENGA - MESTMÄCKER (Hrsg.): Wettbewerbsrecht. Bd. 1, Art 23 VO 1/2003 Rn. 94 ff.

99 BIERMANN: In: IMMENGA - MESTMÄCKER (Hrsg.): Wettbewerbsrecht. Bd. 1, Art 23 VO 1/2003 Rn. 151 ff. 
Die unionsrechtlichen Leitlinien enthalten Öffnungsklauseln, durch die es sich die Kommission vorbehält, beispielsweise von dem eigentlich aufgestellten Rahmen des für die Berechnung der Geldbuße maßgeblichen Ausgangsbetrags von 30\% des mit dem von der Zuwiderhandlung betroffenen Produkts erzielten Umsatzes abzuweichen oder die Geldbuße um einen sog. „Abschreckungsaufschlag“ zu erhöhen. ${ }^{100}$ Nichtsdestotrotz enthalten die Leitlinien eine Selbstbeschränkung des Ermessens der Kommission, die im Regelfall die darin aufgeführten Kriterien beachten muss. Insofern erfordern Abweichungen im Einzelfall eine besondere Begründung, die dem Gleichbehandlungsgrundsatz Rechnung trägt. Aufgrund der vielen offenen Bewertungsfaktoren der Leitlinien 2006 kommt der Kommission bei der Bußgeldbemessung weiterhin ein erheblicher Ermessensspielraum zu, so dass die Leitlinien kein berechtigtes Vertrauen bezüglich der Höhe des Grundbetrags, der Aufschläge und der Endbeträge schaffen. ${ }^{101}$

In der 10. deutschen GWB-Novelle werden die bisherigen Kriterien für die Bußgeldbemessung konkretisiert, indem ein nicht abschließender Kriterienkatalog von bisher schon genutzten Zumessungskriterien kodifiziert wird. Der Gesetzgeber verfolgt damit das Ziel, die Rechtssicherheit bei der Ausfüllung des weiten Bußgeldrahmens zu erhöhen und die kartellbehördliche und gerichtliche Bußgeldbemessung zu harmonisieren - bislang hielt die höchstrichterliche Rechtsprechung die kartellrechtlichen Bußgeldbemessungsrichtlinien für die Rechtsprechung für nicht verbindlich.

Das bislang in der sog. Bonusregelung des Bundeskartellamtes enthaltene Kronzeugenprogramm ist nunmehr - ohne wesentliche Änderungen inhaltlicher Natur gesetzlich verbindlich geregelt. Ungelöst geblieben ist allerdings die Schieflage zwischen kartellbehördlichen und staatsanwaltschaftlichen Ermittlungsverfahren in Kartellfällen: Während für kartellbehördliche Verfahren ein Kronzeugenprogramm zur Verfügung steht, existiert ein solches für ein von der Staatsanwaltschaft geführtes Strafverfahren nicht. Sofern ein Kartellverstoß zugleich ein strafbares Verhalten darstellt, wie dies beim Ausschreibungsbetrug der Fall ist, kann die Kartellbehörde das Verfahren an die Staatsanwaltschaft abgeben, mit der Folge, dass in dem Strafverfahren ein Kronzeugenantrag nur noch im Rahmen der Strafzumessung berücksichtigt werden kann.

\subsection{Vorgaben des Grundsatzes „ne bis in idem“ und des Doppelverfolgungs- verbots vor erstmaliger Sanktionierung in Bezug auf Geldbußen und Kri- minalstrafen}

Geldstrafen sind subsidiär zu Kriminalstrafen, denn die Kriminalstrafe ist das schärfste Sanktionsmittel, das dem Staat zur Verfügung steht, um auf begangenes

${ }^{100}$ Eingehend dazu BIERMANN: In: IMMENGA - MESTMÄCKER (Hrsg.): Wettbewerbsrecht. Bd. 1, Art 23 VO 1/2003 Rn. 220 ff. mit weit. Nachw.

${ }^{101}$ EuG 15.3.2006, T-15/02, Rn. 252, Slg 2006, II-497 (BASF); siehe dazu auch WAGNERVON PAPP, Florian: Best and even better practices in commitment procedures after Alrosa: The dangers of abandoning the "struggle for competition law", Common Market Law Review, 2012, 929 ff. 
Unrecht zu reagieren. Einer doppelten Bestrafung derselben Tat steht der Grundsatz „ne bis in idem“ entgegen, der sich zum einen aus Art. 4 des 7. Zusatzprotokolls zur EMRK und aus Art. 50 Grundrechtecharta entnehmen lässt und der zum anderen in den Mitgliedstaaten der Europäischen Union anerkannt ist. ${ }^{102}$ Außerdem steht das Verbot der Parallelverfolgung, das sich insbesondere aus dem Rechtsstaatsprinzip (Waffengleichheit) ergibt, bereits vor einer erstmaligen rechtskräftigen Sanktionierung der parallelen Ermittlung durch die Wettbewerbsbehörde und die Staatsanwaltschaft entgegen. ${ }^{103} \mathrm{Zu}$ solchen Ermittlungen kommt es insbesondere, wenn dieselbe Tat im prozessualen Sinn sowohl straf- als auch bußgeldbewehrt ist, so z. B. bei Submissionsabsprachen, bei denen die an der Preisabsprache beteiligten juristischen Personen mit einer Kartellgeldbuße und zudem wegen der in Umsetzung der Kartellabsprache abgegebenen Angebote im Rahmen einer Ausschreibung mit einer Verbandsstrafe belegt werden können. In solchen Fällen ist den staatsanwaltschaftlichen Ermittlungen Vorrang vor wettbewerblichen Ermittlungen einzuräumen. ${ }^{104}$

\subsection{Anforderungen an ein rechtsstaatliches Sanktionsverfahren}

Die unionsrechtlichen Geldbußen gegen Unternehmen werden in einem Verwaltungsverfahren verhängt. Hierbei sind die rechtsstaatlichen Garantien, die für kriminalstrafrechtliche und strafrechtliche Sanktionen im weiteren Sinne wie Geldbußen Geltung beanspruchen, zu berücksichtigen. Allerdings war der EuGH bei der Anerkennung solcher Garantien im Zusammenhang mit Geldbußen speziell gegen Unternehmen zurückhaltend, hat allerdings in der Rechtssache „Menarini Diagnostics“ entschieden, dass (italienische) Kartellbußen als strafrechtliche Anklage im Sinne der Konvention zu bewerten seien. ${ }^{105}$ Dies gilt gleichermaßen für den deutschen Gesetzgeber, der sich diesbezüglich am EU-Recht orientiert, wie ein Blick auf die zehnte Novelle des Gesetzes gegen Wettbewerbsbeschränkungen zeigt. ${ }^{106}$ Dieses Gesetz bringt einschneidend Veränderungen der deutschen Rechtslage im Bereich der Mitwirkungs- und Offenlegungspflichten Betroffener mit sich, die unter rechtsstaatlichen Gesichtspunkten bedenklich sind:

Der Betroffene eines Kartellbußgeldverfahrens hat nur noch ein sehr eingeschränktes Schweigerecht inne. In diesem Punkt wird die sog. Orkem-Rechtsprechung des $\mathrm{EuGH}^{107}$ kodifiziert: Hiernach darf der Betroffene zwar nicht zu einem

102 Eingehend dazu DANNECKER, Gerhard: Ne bis in idem und das Verbot straf- und kartellrechtlicher Parallelverfahren. Wien, 2021, 19, 21 ff., $105 \mathrm{f}$.

103 DANNECKER (2021): a.a.O. 75 ff., 107.

104 Eingehend dazu DANNECKER (2021): 97 ff., 108.

105 EuGH, 27. 09. 2011, Rs. C-43509/08, Rn. 44

106 „Gesetz zur Änderung des Gesetzes gegen Wettbewerbsbeschränkungen für ein fokussiertes, proaktives und digitales Wettbewerbsrecht 4.0 und anderer wettbewerbsrechtlicher Bestimmungen“ („GWB-Digitalisierungsgesetz“); dazu BIEN, Florian et al. (Hrsg.): Die 10. GWB-Novelle: Das neue Kartellrecht. München, 2021.

${ }^{107}$ EuGH, 18.05.1989, Rs. C-374/87, Slg. 1989, 3283. 
Geständnis einer Zuwiderhandlung gegen Art. 101 bzw. 102 AEUV gezwungen werden, allgemeine Fragen zu Umständen einer Zuwiderhandlung, aus denen im Wege eines Indizienbeweises auf die Zuwiderhandlung geschlossen werden kann, sind aber zulässig. Damit geht eine Senkung des bislang geltenden deutschen Schutzniveaus auf das niedrigere EU-Schutzniveau einher.

Das Gesetz konkretisiert die Kriterien für die Bußgeldbemessung und schafft damit gesetzlich bestimmtere Maßstäbe. Damit ist ein nicht abschließender Kriterienkatalog von bisher schon genutzten Zumessungskriterien kodifiziert. Der Gesetzgeber strebt damit ein Mehr an Rechtssicherheit bei der Ausfüllung des weiten Bußgeldrahmens an sowie eine Harmonisierung der kartellbehördlichen und gerichtlichen Bußgeldbemessung. Bisher haben die Gerichte die behördlichen Sentencing Guidelines als nur für die Verwaltungsbehörde verbindlich angesehen und die Bußgeldbemessung losgelöst von diesen Vorgaben nach eigenen Kriterien vorgenommen.

\section{FAZIT}

Die Entwicklung des unionsrechtlichen Unternehmensbußgeldrechts und der nationalen Verbandsstrafen aufeinander zu ist eine durchaus erfreuliche Entwicklung, die aber noch eine Reihe von Problemen, insbesondere bezüglich der Sanktionsbemessung und der Verfahrensgarantien, mit sich bringt. Der sich abzeichnende Weg der Konvergenz trotz unterschiedlicher Sanktionsmodelle - Unternehmens- versus Verbandsverantwortlichkeitsmodell - sollte angesichts der Strafähnlichkeit der Geldbuße fortgesetzt werden. Hierbei ist es unverzichtbar, den rechtsstaatlichen Anforderungen an das materielle Sanktionsrecht als auch an das Verfahrensrecht zu entsprechen und die verantwortlichen Unternehmen als „,corporate citizens” anzuerkennen. ${ }^{108}$

\section{LITERATURVERZEICHNIS}

[1] ACHENBACH, Hans: Die ECN+-Richtlinie (EU) 2019/1 und das deutsche Kartellordnungswidrigkeitenrecht. wistra, 2019, $257 \mathrm{ff}$.

[2] AlbRECHT, Jan Philipp: Das neue EU-Datenschutzrecht - von der Richtlinie zur Verordnung. CR, 2016, $88 \mathrm{ff}$.

[3] AlBRECHT, Jan Philipp - JotZO, Florian: Das neue Datenschutzrecht der EU. 2017.

[4] BAUER, Raphaela: Der Allgemeine Teil des Kartellbußenrechts. Wien, 2020.

[5] BEHR, Nicolai - TANNEN, Florian: Droht das Zeitalter der Datenschutzgeldbußen? ... und was Unternehmen dagegen tun können. CCZ, 2020, $120 \mathrm{ff}$.

${ }^{108}$ Eingehend dazu DANNECKER, Gerhard - SCHRÖDER, Thomas: Overcoming the current system of corporate criminal law - sanctioning corporate citizens. Crime prevention within the limits of rule the of law. In: POHLMANN, Markus - DANNECKER, Gerhard VALARINI, Elizangela (eds.): Bribery, Fraud, Cheating. How to Explain and to Avoid Organizational Wrongdoing, 2020, $285 \mathrm{ff}$. 
[6] Bergt, Matthias: Datenschutz und Datensicherheit. DuD, 2017, 555.

[7] BIEN, Florian et al. (Hrsg.): Die 10. GWB-Novelle: Das neue Kartellrecht. München 2021.

[8] CORNELIUS, Kai: Die „datenschutzrechtliche Einheit“ als Grundlage des bußgeldrechtlichen Unternehmensbegriffs nach der EU-DSGVO. NZWiSt 2016, 421.

[9] DANNECKER, Christoph: Konturierung prozessualer Gewährleistungsgehalte des nemo tenetur- Grundsatzes anhand der Rechtsprechung des EGMR. ZStW, 127 (2015), $370 \mathrm{ff}$.

[10] DANNECKER, Gerhard: Die Sanktionierung von Verstößen gegen das gemeinschaftsrechtliche Kartellrecht nach der VO (EG) Nr. 1/2003, wistra, 2004, $361 \mathrm{ff}$.

[11] DANNECKER, Gerhard: Die Neuregelung der Sanktionierung von Verstößen gegen das EG-Kartellrecht nach der Verordnung (EG) Nr. 1/2003 des Rates vom 16. Dezember 2002 zur Durchführung der in den Art. 81 und 82 des Vertrages niedergelegten Wettbewerbsregeln. In: Festschrift für Ulrich Immenga zum 70. Geburtstag. München, 2004, $61 \mathrm{ff}$.

[12] DANNECKER, Gerhard: Der strafrechtliche Schutz des Wettbewerbs: Notwendigkeit und Grenzen einer Kriminalisierung von Kartellrechtsverstößen. In: Festschrift für Klaus Tiedemann zum 70. Geburtstag. Köln, 2008, 789 ff.

[13] DANNECKER, Gerhard: Stellungnahme zum Zwischenbericht des Bundeskartellamts zum Expertenkreis Kartellsanktionenrecht. NZKart, 2015, p. 14.

[14] DANNECKER, Gerhard: Strafe oder bloße Ordnungswidrigkeit - europäische Vorgaben und Entwicklungen. In: LEITNER, Roman - BRANDL, Rainer (Hrsg.): Finanzstrafrecht 2016. Wien, 2017, $125 \mathrm{ff}$.

[15] DANNECKER, Gerhard: Das Kartellordnungswidrigkeitenrecht im Wandel: Von der Verbands- zur Unternehmensverantwortung? In: Festschrift für Rudolf Rengier zum 70. Geburtstag. München, 2018, $193 \mathrm{ff}$.

[16] DANNECKER, Gerhard: Grundstrukturen des Grundsatzes „ne bis in idem“ im Spiegel der Rechtsprechung des Europäischen Gerichtshofs für Menschenrechte und des Europäischen Gerichtshofs. In: Festschrift für Ákos Farkas zum 65. Geburtstag. Bd. 1, 2019, 206 ff. (https://www.mjsz.uni-miskolc.hu/files/6553/23_danneckergerhard_t $\%$ C3\%Bórdelt.pdf).

[17] DANNECKER, Gerhard: Ne bis in idem und das Verbot straf- und kartellrechtlicher Parallelverfahren. Wien, 2021.

[18] DANNECKER, Gerhard - DANNECKER, Christoph: Europäische und verfassungsrechtliche Vorgaben für das materielle und formelle Unternehmensstrafrecht. NZWiSt, 2016, 162 ff. 
[19] DANNECKER, Gerhard - DANNECKER, Christoph: Kartellsanktionsrecht. In: SOYER, Richard (Hrsg.): Handbuch Unternehmensstrafrecht. Wien, 2021.

[20] DANNECKER, Gerhard - FISCHER-FRITSCH, Jutta: Das EG-Kartellrecht in der Bußgeldpraxis. 1989.

[21] DANNECKER, Gerhard - KÖRTEK, Jasemin: General Report. In: DANNECKER, Gerhard - JANSEN, Oswald (eds.): Competition Law Sanctioning in the European Union. 2004, $1 \mathrm{ff}$.

[22] DANNECKER, Gerhard - MÜLLER, Nadja: Das Kartellsanktionenrecht im Lichte der 9. GWB-Novelle. $K S z W, 2017,125$ ff.

[23] DANNECKER, Gerhard - DANNECKER, Christoph - MÜLlER, Nadja: Das Kartellordnungswidrigkeitenrecht nach der 8. GWB-Novelle: weiterer Re- formbedarf? ZWeR, 2013, $417 \mathrm{ff}$.

[24] DANNECKER, Gerhard - SCHRÖDER, Thomas: Tatbestände mit supranationaler Schutzrichtung (Europadelikte). In: BÖSE, Martin (Hrsg.): Europäisches Strafrecht (EnzEuR Bd. 11). 2, Aufl., 2021.

[25] DANNECKER, Gerhard - SCHRÖDER, Thomas: Overcoming the current system of corporate criminal law - sanctioning corporate citizens. Crime prevention within the limits of rule the of law. In: POHLMANN, Markus - DANNECKER, Gerhard - VAlARINI, Elizangela (eds.): Bribery, Fraud, Cheating. How to Explain and to Avoid Organizational Wrongdoing. 2020, $285 \mathrm{ff}$.

[26] EhMANN, Eugen - SELMAYR, Martin: Datenschutz-Grundverordnung: DSGVO. Münchenm, 2. Aufl., 2018.

[27] ESSER, Robert: Das Doppelverfolgungsverbot in der Rechtsprechung des EGMR Das Doppelverfolgungsverbot in der Rechtsprechung des EGMR (Art. 4 des 7. ZP EMRK). Divergenzen und Perspektiven. In: HOCHMAYR, Gudrun (Hrsg.): Ne bis in idem in Europa. Baden-Baden, 2015, $27 \mathrm{ff}$.

[28] FedermanN, Bernd A.: Kriminalstrafen im Kartellrecht. 2006.

[29] FAUST, Sebastian - SPITTKA, Jan - WYBITUL, Tim: Milliardenbußgelder nach der DS-GVO? ZD, 2016, 120.

[30] GRÜNWALD, Andreas - HACKL, Jens: Das neue umsatzbezogene Sanktionsregime der DS-GVO. ZD, 2017, $556 \mathrm{ff}$.

[31] HEINICHEN, Christian: Unternehmensbegriff und Haftungsnachfolge im europäischen Kartellrecht. Baden-Baden, 2011.

[32] HENN, Florian: Strafrechtliche Verfahrensgarantien im europäischen Kartellrecht. Implikationen und Grenzen der Strafrechtsähnlichkeit von Kartellbußgeldern. 2018, 33. 
[33] HoppmanN, Erich: Das Konzept der optimalen Wettbewerbsintensität, JbNSt, 179 (1966), 286, 289 und 300.

[34] IMMENGA, Ulrich - MESTMÄCKER, Ernst Joachim (Hrsg.): Wettbewerbsrecht. Bd. 1, 6. Aufl. 2020.

[35] KROHM, Niclas: Die wirtschaftliche Einheit als Bußgeldadressat unter der Datenschutz-Grundverordnung? RDV, 2017, 221.

[36] KÜHLING, Jürgen - BUCHNER, Benedikt: Datenschutz-Grundverordnung, Bundesdatenschutzgesetz: DS-GVO/BDSG. 3. Aufl., 2020.

[37] VOn LAUfEnBERG, Christoph: Kartellrechtliche Konzernhaftung, Berlin 2018.

[38] LESSENICH, Christof A.: Unternehmensbegriff und Zurechnung. Bonn 2000.

[39] LoewENHEIM, Ulrich - MEeSEN, Karl M. - RIESENKAMPFF, Alexander KERSTING, Christian - MEYER-LindEMANN, Hans Jürgen (Hrsg.): Kartellrecht. Kommentar zum Deutschen und Europäischen Recht. 4. Aufl., 2020, München.

[40] LUTZ, Martin: Amnestie für aufklärungsbereite Kartellanten?, BB, 2000, 677 ff.

[41] MAUNZ, Theodor/DÜRIG, Günter (Hrsg.): Grundgesetz. Kommentar, 62. Ergänzungslieferung. 2011.

[42] MITSCH, Wolfgang (Hrsg.): Karlsruher Kommentar zum Gesetz über Ordnungswidrigkeiten. 5. Aufl., 2018.

[43] MöHLENKAMP, Andreas: Die europäische Bußgeldpraxis aus Unternehmenssicht in: SCHWARZE, Jürgen (Hrsg.): Instrumente zur Durchsetzung des europäischen Wettbewerbsrechts. 2002.

[44] Ost, Konrad: Die Richtlinie 1/2019: Ein Meilenstein für die Rechtsdurchsetzung im European Competition Network. NZKart, 2019, 69.

[45] PaAl, Boris - Pauly, Daniel A. - Frenzel, Eike Michael: DatenschutzGrundverordnung. 2. Aufl., 2018.

[46] Plath, Kai Uwe (Hrsg.): DSGVO/BDSG. 3. Aufl., 2018.

[47] RAUE, Frank: Müssen Grundrechtsbeschränkungen wirklich verhältnismäßig sein? Archiv des öffentlichen Rechts, 131 (2006), 79 ff.

[48] RIZVI, Salim: Entfesselte Bussenpraxis im Wettbewerbsrecht? AJP/PJA, $2010,452$.

[49] SCHILDGEN, Larissa: Rechtsfähigkeit des Unternehmens im Unionswettbewerbsrecht. 2020, $7 \mathrm{ff}$.

[50] ScHÖNEFELD, Jana - THOMÉ, Sarah: Auswirkungen der Datenschutz-Grundverordnung. PinG, 2017, 126.

[51] SCHULTE, Lothar - JUST, Christoph: Kartellrecht. Art. 23 VO 1/2003 Rz 40 ff. 
[52] STUCKENBERG, Carl-Friedrich: $§ 10$ Allgemeiner Teil eines Europäischen Strafrechts. In: BÖSE, MARTIN (Hrsg.): Europäisches Strafrecht (EnzEuR Bd. 11). 2. Aufl., 2021.

[53] TIEDEMANN, Klaus: Der Allgemeine Teil des europäischen supranationalen Strafrechts. In: Festschrift für Hans-Heinrich Jescheck zum siebzigsten Geburtstag. 1985.

[54] Tiedemann, Klaus: Wirtschaftsstrafrecht. München, 5. Aufl., 2017.

[55] UeBELE, Fabian: Das „Unternehmen“ im europäischen Datenschutzrecht. $E u Z W, 2018,440$.

[56] VenN, Nikolai - WyBITUL, Tim: Die bußgeldrechtliche Haftung von Unternehmen nach Art. 83 DS-GVO (zugl. Anm. zu LG Bonn Urt. v. 11. 11. 2020 - 29 OWi 1/20). NStZ, 2021, $204 \mathrm{ff}$.

[57] WAGNER VON PAPP, Florian: Kartellstrafrecht in den USA, dem Vereinigten Königreich und Deutschland. WuW, 2009, 1236 ff.

[58] WAGNER-VON PAPP, Florian: Best and even better practices in commitment procedures after Alrosa: The dangers of abandoning the "struggle for competition law". Common Market Law Review, 2012, 929 ff.

[59] Wegner, Carsten: Keine umfassende Begründungspflicht der Kommission für Geldbußen in Millionenhöhe? WuW, 2001, 469, 476.

[60] WILS, Wouter P. J.: World Competition. 2005.

[61] WOLFF, Heinrich Amadeus - BRINK, Stefan (Hrsg.): BeckOK Datenschutzrecht. 33. Ed. 1. 8. 2020.

[62] WYBITUL, TIM: EU-Datenschutz-Grundverordnung. 2017. 